\title{
Near-Infrared Imaging of the Central Regions of Metal-Poor Inner Spheroid Globular Clusters
}

\author{
T. J. Davidge ${ }^{1}$ \\ Canadian Gemini Office, Herzberg Institute of Astrophysics, \\ National Research Council of Canada, 5071 W. Saanich Road, \\ Victoria, British Columbia, Canada V9E 2E7 \\ email:tim.davidge@nrc.ca
}

\begin{abstract}
$J H K$ images obtained with the Canada-France-Hawaii Telescope (CFHT) Adaptive Optics Bonnette (AOB) are used to investigate the near-infrared photometric properties of red giant branch (RGB) and horizontal branch (HB) stars in eight metal-poor globular clusters with $\mathrm{R}_{G C} \leq 2 \mathrm{kpc}$. The slope of the RGB on the $(K, J-K)$ CMDs confirms the metal-poor nature of these clusters, four of which (NGC 6287, NGC 6293, NGC 6333, and NGC 6355) have metallicities that are comparable to M92. The luminosity functions of RGB stars in inner spheroid and outer halo clusters have similar slopes, although there is a tendency for core-collapsed clusters to have slightly flatter luminosity functions than non-collapsed clusters. The distribution of red HB stars on the $(K, J-K)$ CMDs of inner spheroid clusters with $[\mathrm{Fe} / \mathrm{H}] \sim-1.5$ is very different from that of clusters with $[\mathrm{Fe} / \mathrm{H}] \sim-2.2$, suggesting that metallicity is the main parameter defining $\mathrm{HB}$ content among these objects. The RGB-bump is detected in four of the inner spheroid clusters, and this feature is used to compute distances to these objects. Finally, the specific frequency of globular clusters in the inner Galaxy is discussed in the context of the early evolution of the bulge. Based on the ratio of metal-poor to metal-rich clusters in the inner Galaxy it is suggested that the metal-poor clusters formed during an early intense burst of star formation. It is also demonstrated that if the globular cluster formation efficiency for the inner Galaxy is similar to that measured in other spheroidal systems, then the main body of the bulge could have formed from gas that was chemically enriched in situ; hence, material from a separate pre-enriched reservoir, such as the disk or outer halo, may not be required to form the bulge.
\end{abstract}

\footnotetext{
${ }^{1}$ Visiting Astronomer, Canada-France-Hawaii Telescope, which is operated by the National Research Council of Canada, the Centre National de la Recherche Scientifique de France, and the University of Hawaii
} 
Subject headings: globular clusters:general - Galaxy: center - infrared:stars stars: late type - stars: giant branch

\section{INTRODUCTION}

As the brightest members of the inner spheroid, globular clusters provide an important means of probing the structure and early evolution of the inner Galaxy. The most metal-rich inner spheroid clusters likely formed as part of the bulge (Minniti 1995a, Côté 1999), and other sub-structures (Burkert \& Smith 1997), although some may belong to the classical halo (Heitsch \& Richtler 1999). Minniti (1996) argues that the metal-poor globular clusters in the central regions of the Galaxy are part of the classical halo, although the orbital properties of these objects are similar to those of bulge stars and gas in the inner Galaxy (Côté 1999). In addition, globular clusters in the inner Galaxy are more compact than those in the outer halo (van den Bergh 1994). It remains to be determined if the differences in orbital properties and sizes between metal-poor clusters in the inner spheroid and outer halo are primordial or the result of evolution.

Efforts to study globular clusters in the inner spheroid are confronted with formidable observational challenges. Observations at visible wavelengths are complicated by the large, often non-uniform, extinction originating in the disk. The effects of this extinction can be reduced by observing at wavelengths longward of $1 \mu \mathrm{m}$, although then the temperature sensitivity of broad-band colors is decreased for all but the reddest stars. Contamination from disk and bulge stars poses a problem for studies at all wavelengths, and the failure to identify non-cluster stars can bias metallicity and distance estimates (Davidge 2000a,b). Lacking spectroscopic information, contamination from non-cluster sources can be minimized by observing the dense central regions of clusters. While crowding then becomes an issue, stars at the main sequence turn-off (MSTO) can be resolved in the central regions of clusters at near-infrared wavelengths with 4 metre-class telescopes (Davidge \& Courteau 1999).

Davidge (2000a) obtained broad and narrow-band near-infrared images of all known (as of 1996) globular clusters with $[\mathrm{Fe} / \mathrm{H}] \leq-1.3$ and $\mathrm{R}_{G C}<3 \mathrm{kpc}$, and used the properties of the brightest giants to compute the metallicities, reddenings, and distances of these objects. In the present study, $J H K$ images obtained with the CFHT AOB are used to investigate the photometric properties of stars as faint as the sub-giant branch (SGB) in eight metal-poor inner spheroid clusters, selected to have $[\mathrm{Fe} / \mathrm{H}]<-1$ and $\mathrm{R}_{G C}<2 \mathrm{kpc}$. 
The clusters are listed in Table 1, along with key properties taken from the June 1999 compilation of Harris (1996); in most cases the metallicities are from Zinn \& West (1984). The structural designations in the last column are from Trager, King, \& Djorgovski (1995). The properties of three outer halo clusters, spanning most of the metallicity range of the inner spheroid sample, and which are used as a control sample, are also listed in Table 1. The data for NGC 6287, M13, and M92 used in the current study were discussed previously by Davidge \& Courteau (1999).

Many of the inner spheroid clusters listed in Table 1 have been observed previously in the infrared. Minniti, Olszewski, \& Rieke (1995) presented $(K, J-K)$ color-magnitude diagrams (CMDs) of NGC 6287, NGC 6293, NGC 6333, NGC 6401, NGC 6522, and NGC 6642, while Davidge (2000a) presented $(K, J-K)$ CMDs and $K$ LFs covering the upper giant branch of all of the inner spheroid clusters in Table 1 except NGC 6401. The data discussed in the current paper go much fainter than these earlier studies; not only does this allow for a more detailed investigation of the properties of the RGB, which can be used to refine metallicity and distance estimates, but it also enables a preliminary reconnaisance of the cluster HBs.

The paper is structured as follows. The observations and the data reduction procedures are described in $\S 2$, while the photometric measurements, CMDs, HB properties, giant branch LFs, and RGB-bump measurements are discussed in $\S 3-6$. A brief discussion of the results follows in $\S 7$.

\section{OBSERVATIONS \& REDUCTIONS}

The data were obtained with the CFHT AOB (Rigaut et al. 1998) and KIR camera during the nights of UT September 5, 6, and 7 1998. KIR contains a $1024 \times 1024 \mathrm{Hg}$ :Cd:Te array, with each pixel subtending 0.034 arcsec on a side when used with the AOB.

AO reference stars were selected at the telescope based on brightness, the absence of bright companions, and proximity to the cluster center. Once selected, the guide stars were centered on the detector to minimize the effects of anisoplanicity near the edge of the science field. In some cases this repositioning caused the cluster center to be offset to one quadrant of the detector.

Each cluster was observed through $J, H$, and $K s$ filters. The telescope was offset between individual exposures in a $0.5 \times 0.5$ arcsec square dither pattern to facilitate the suppression of bad pixels and the construction of calibration frames from on-sky images. The main dataset for each cluster consists of exposures with $90 \mathrm{sec}$ integration times 
recorded at each dither position. However, in many cases the brightest stars were saturated, so a second series of images with $3-5$ sec integration times were also recorded.

Standard stars from Casali \& Hawarden (1992) were also observed during the course of the three night observing run. The uncertainty in the photometric zeropoint of each filter derived from these observations is \pm 0.03 mag.

Deep exposures of NGC 6287 were obtained by Davidge \& Courteau (1999), and so only images with $5 \mathrm{sec}$ integration times per dither position were recorded of this cluster during the September 1998 run. An AO reference star located 14 arcsec away from the reference star used by Davidge \& Courteau (1999) was selected so that the effects of anisoplanicity on the photometric measurements could be assessed (§3); details of the deep NGC 6287 observations, as well as the M13 and M92 observations that are also discussed in this paper, can be found in Davidge \& Courteau (1999).

The data were reduced using the procedures described by Davidge \& Courteau (1999). The basic steps in the reduction process were: (1) subtraction of dark frames, (2) division by flat-field frames, and (3) the subtraction of thermal signatures and the DC sky level. The results were aligned to correct for the offsets introduced during acquisition, and then median-combined. The final $K$ images of the inner spheroid clusters observed during the September 1998 run are shown in Figures 1 and 2. In most cases the image quality is between 0.2 and 0.3 arcsec FWHM, and conspicuous signatures of anisoplanicity, such as the elongation of stellar images near the field edge (e.g. McClure et al. 1991), are not apparent.

\section{PHOTOMETRIC MEASUREMENTS}

A single point-spread function (PSF) was constructed for each image using tasks in the DAOPHOT (Stetson 1987) photometry package, and stellar brightnesses were then measured with the PSF-fitting program ALLSTAR (Stetson \& Harris 1988). The finite size of atmospheric turbulence cells causes the PSF to vary with distance from the reference star ('anisoplanicity') on angular scales of a few arcsec, and so the use of a single PSF introduces

position-dependent photometric errors. While it would be preferable to construct a variable PSF that tracks the affects of aniosplanicity, crowding and the complicated nature of the PSF variation with distance from the reference source make it difficult to do so. In any event, previous studies of globular clusters with the AOB indicate that anisoplanicity does not introduce photometric uncertainties larger than a few percent over angular scales comparable to that of the KIR science field (Davidge \& Courteau 1999; Davidge 2000b). 
The effects of anisoplanicity can be investigated by comparing stellar brightnesses measured from images of a field recorded using different $\mathrm{AO}$ reference stars. The $K$ images of the March and September 1998 NGC 6287 datasets in the vicinity of the reference stars are compared in Figure 3. The PSF changes most rapidly with location close to the reference stars, and so a sample of moderately bright stars, which are marked in Figure 3, were selected to assess the impact of PSF variations on the photometry.

Anisoplanicity will cause the difference in $K$ brightness, $\Delta K$, between the measurements from the March 1998 and September 1998 datasets to vary across the field. The two reference stars are separated by roughly 14 arcsec, and in the top two panels of Figure 4 the histogram distribution of $\Delta K$ values for stars located within 7 arcsec of the September 1998 reference star (interval 1) is compared with the distribution of stars located between 7 and 14 arcsec from this star (interval 2). The mean $\Delta K$ for all stars is $0.03 \pm 0.03$, confirming that the two datasets have consistent photometric calibrations. The short exposure time of the September 1998 dataset introduces scatter that is significant for the faintest sources used in this comparison, which have $K=14$; artificial star experiments (see below) indicate that the uncertainties introduced by crowding and noise at this brightness in the September 1998 dataset is $\pm 0.1 \mathrm{mag}$. The relation between $K$ and $\Delta K$ is shown in the lower panel of Figure 4, and it is evident that the stars defining the two extremes in $\Delta K$ are among the faintest in the comparison. Photometric variability is another potential source of scatter.

The mean values of $\Delta K$ in the two radial intervals are not significantly different: $\overline{\Delta K}=-0.02 \pm 0.04$ in interval 1 , and $\overline{\Delta K}=0.06 \pm 0.03$ in interval 2 . While based on only a modest sample of stars, this comparison suggests that anisoplanicity introduces uncertainties of no more than a few percent in the $K$ photometric measurements of sources near the reference star, as originally suggested by Davidge \& Courteau (1999).

Artificial star experiments were used to assess sample completeness and estimate the effects of crowding and random noise on the photometry. Artificial stars were added to each final image using the ADDSTAR routine in DAOPHOT, which scales the PSF constructed for each dataset and adds random noise. The number of added stars was restricted to a small fraction of the number of stars actually detected to prevent artificially increasing the amount of crowding. Because a fixed PSF is assumed, these experiments do not monitor uncertainties introduced by anisoplanicity; however, the scatter in the CMDs is consistent with that predicted from the artificial star experiments $(\S 4)$, indicating that anisoplanicity is not the dominant source of noise.

The artificial star experiments indicate that the long exposure $K$ data are typically complete to $K=14$, with $50 \%$ completeness occuring at $K=17$. The photometric 
uncertainties are typically $\pm 0.02-0.04 \mathrm{mag}$ at $K=14$, and $\pm 0.2-0.3$ mag at $K=17$.

\section{COLOR-MAGNITUDE DIAGRAMS}

The $(K, H-K)$ and $(K, J-K)$ CMDs of the inner spheroid clusters are shown in Figures 5 and 6 , while the corresponding CMDs for the outer halo clusters are plotted in Figures 7 and 8 . The photometry for stars brighter than $K=12$ was obtained from the short exposure images. Images with short exposure times were not recorded for M92, and so the bright cut-off for this cluster is defined by detector saturation.

The main sequence turn-off (MSTO) for old metal-poor systems occurs near $\mathrm{M}_{K}=3$ (Bertelli et al. 1994), which corresponds to $K=17.5-18$ at the distance of the Galactic Center (Reid 1993; Davidge 2000a). Hence, the CMDs of the inner spheroid clusters, which typically extend to $K=18$, sample the entire giant branch, including the SGB, although incompleteness becomes significant at this point ( $(5)$. The CMDs for NGC 6287 are deeper than those of the other clusters because the data were recorded on a night with significantly better image quality.

The $H-K$ color is insensitive to effective temperature, and so the width of the $(K, H-K)$ CMD provides an independent means of assessing star-to-star photometric scatter. The standard deviation about the mean $H-K$ value in Figure 5 for stars with $K$ between 12 and 14 is typically \pm 0.02 to \pm 0.03 magnitude, suggesting that the random uncertainty in individual photometric measurements is on the order of \pm 0.02 magnitudes. This is consistent with the uncertainties predicted by the artificial star experiments, which do not account for PSF variations across the field, thus suggesting that anisoplanicity is not the dominant source of photometric scatter.

Kuchinsky et al. (1995) investigated the relation between metallicity and the slope of the giant branch on $(K, J-K)$ CMDs, and the calibration of this relation has recently been extended to very metal-poor clusters by Ferraro et al. (2000) and Ivanov et al. (2000). The slope of the upper giant branch was measured from the $(K, J-K)$ CMDs in Figures 6 and 8 using the procedures described by Kuchinsky et al. (1995), and the results are listed in the second column of Table 2.

The giant branch slopes of NGC 6287, NGC 6293, NGC 6333, and NGC 6355 are similar to M92, confirming that these clusters are very metal-poor. The metallicities derived from the relation between $[\mathrm{M} / \mathrm{H}]$ and giant branch slope specified in Figure 12 of Ferraro et al. (2000) are listed in the third column of Table 2, and for most of the clusters there is general agreement with the values listed in Table 1. Only a few stars define the giant branch 
sequence of NGC 288, and the central regions of this cluster are also devoid of the brightest giants that have a significant impact on slope measurements (Davidge 2000a); consequently, the RGB slope measured for NGC 288 from the current data is not well defined, and so a metallicity was not calculated.

The HB and RGB sequences tend to be well separated on the $(K, J-K)$ CMDs. Only a modest number of HB stars are detected in each of the outer halo clusters, while significantly richer HB populations are found in the inner spheroid clusters; such a difference in stellar density is not unexpected given that inner spheroid clusters are more compact than outer halo clusters (van den Bergh 1994). The current data can be used to probe the HB content of the inner spheroid clusters, although the scatter in the CMDs becomes significant when $K=17$, and this hinders efforts to study the bluest HB stars; for example, the extremely blue HB stars in NGC 6273 studied by Piotto et al. (1999) will have $K=20-21$.

Searle \& Zinn (1978) compared the HB morphologies of globular clusters in different regions of the Galaxy, and concluded that while the HB morphology of clusters in the inner Galaxy is defined by metallicity, this is not the case for clusters in the outer halo; more recently, the apparent absence of a second parameter affect among clusters in the inner Galaxy has been supported by Lee, Demarque, \& Zinn (1994). Given that age is one of the many (e.g. Salaris \& Weiss 1997, Buonanno et al. 1997) parameters that can affect HB content, then one possible explanation of these results is that the inner Galaxy formed over a shorter period of time than the outer halo, as might be expected if the Galaxy formed from the inside out (e.g. Larson 1990). However, recent studies of globular clusters at very low Galactic latitudes challenge the long-held notion that HB morphology depends solely on metallicity within a few kpc of the Galactic Center (Davidge \& Courteau 1999, Rich et al. 1998; Ortolani et al. 1999).

In an effort to investigate qualitatively the effect of metallicity on HB morphology among metal-poor inner spheroid clusters, composite CMDs were constructed by grouping the clusters according to metallicity, and then combining the CMDs of the clusters in each group. Two groups were considered: moderately metal-poor (NGC 6273, NGC 6401, NGC 6522, and NGC 6642), and very metal-poor (NGC 6287, NGC 6293, NGC 6333, and NGC 6355). The HBs of NGC 6273 and NGC 6522 in Figure 6 are not well-defined, and so a composite CMD for moderately metal-poor clusters that did not include these objects was also constructed.

The CMDs in each group were corrected for cluster-to-cluster differences in reddening by registering the CMDs along the horizontal axis using the mean giant branch color at $K=14$ as a reference. A corresponding shift to the vertical axis based on the Rieke \& 
Lebofsky (1985) extinction curve was also applied. The inner spheroid clusters are roughly equidistant, so no effort was made to adjust for differences in distance.

The composite CMDs are compared in Figure 9, and the $\mathrm{HB}$ in the $[\mathrm{Fe} / \mathrm{H}]=-2$ $\mathrm{CMD}$ is clearly different from the $[\mathrm{Fe} / \mathrm{H}]=-1.5$ CMDs computed with or without NGC 6273 and NGC 6522. In particular, the HB of the moderately metal-poor group consists of stars that are more or less uniformly distributed to the left of the giant branch with $\Delta K$ between 2 and 0. For comparison, the HB of the very metal-poor group is dominated by a clump of stars with a J-K color that is roughly 0.5 mag bluer than the giant branch. This simple comparison indicates that metallicity is the main parameter defining red $\mathrm{HB}$ content among metal-poor inner spheroid clusters, although the possibility that other parameters affect $\mathrm{HB}$ content of course can not be ruled out.

\section{GIANT BRANCH LUMINOSITY FUNCTIONS}

The $K$ LFs of RGB stars, which were constructed from the CMDs after removing HB stars, are plotted in Figures 10 (inner spheroid clusters) and 11 (outer halo clusters). The LFs follow power-laws, and so can be characterized by an exponent, $x$, which was measured for each cluster by using the method of least squares to fit a power-law to the completeness-corrected data between $K=12$ and $16 . K=12$ was selected as the bright limit to avoid small number statistics near the RGB-tip, while $K=16$ was adopted as the faint limit to avoid the onset of the sub-giant branch (SGB), which the models of Bertelli et al. (1994) predict should occur between $K=16$ and 17 in old stellar systems at the distance of the Galactic Center (GC).

The $x$ values for the various clusters are listed in the second column of Table 3 . Davidge (2000a) measured LF exponents for many of these clusters using images that, while covering a much larger area, sampled only the brightest cluster members, and his $x$ values are listed in the last column of Table 3 . The mean difference between the two sets of exponents in Table 3 is $\Delta x=0.05 \pm 0.04$, indicating general agreement. However, significant differences occur for some clusters. For example, in the case of NGC 6287, which Davidge (2000a) noted would have a markedly different exponent if stars fainter than $K=12.5$ were included, $\Delta x=-0.16 \pm 0.04$.

The unweighted mean exponent for the eight inner spheroid clusters is $\bar{x}=0.31 \pm 0.02$, which is not significantly different from the mean of the three outer halo clusters, which is $\bar{x}=0.29 \pm 0.03$. Therefore, metal-poor clusters in the inner spheroid and outer halo have, on average, similar LF exponents. Nevertheless, the uncertainties in $x$ are typically 
$\pm 0.02-0.04$, and the entries for the inner spheroid clusters in Table 3 span a much larger range than this, suggesting that real cluster-to-cluster scatter might be present. Three of the inner spheroid clusters are core-collapsed, and the central regions of core-collapsed clusters tend to be devoid of bright giants (e.g. Djorgovski et al. 1991; Janes \& Heasley 1991; Djorgovski \& Piotto 1993; Davidge 1995, Burgarella \& Buat 1996). The absence of bright stars might be expected to affect the LF exponents, and hence introduce cluster-to-cluster scatter in the $x$ measurements - is evidence for this seen in the current data?

There is a remarkable degree of uniformity among the $x$ values for the core-collapsed clusters in this sample, with $\bar{x}=0.27 \pm 0.01$ for NGC 6293, NGC 6355, and NGC 6522. For comparison, the unweighted mean of the remaining five inner spheroid clusters is $\bar{x}=0.34 \pm 0.03$, while $\bar{x}=0.32 \pm 0.02$ if the three outer halo clusters are also considered. These data hint that there may be a slight difference between the LF exponents of dynamically evolved and unevolved clusters, although there is clearly a need to sample a larger number of objects to confirm these results. The sense of the difference in $x$ between the dynamically evolved and unevolved clusters is somewhat surprising, as the absence of bright giants would be expected to produce a steeper LF, which is contrary to what is seen here. However, Davidge (1991) compared the optical and near-infrared color profiles of the globular cluster NGC 4147, which Djorgovski \& King (1986) suggest might be core-collapsed, and concluded that the giant branch population near the cluster center is depleted at brightnesses well below the RGB-tip, extending at least as faint as the HB; consequently, the depletion of stars near the RGB-tip might only be the most conspicuous signature of a process that occurs over a larger range of brightnesses.

\section{THE RGB-BUMP}

As a star ascends the giant branch the hydrogen-burning shell encounters a discontinuity in the abundance profile that marks the maximum inward extent of envelope convection (e.g. Iben 1968). The pace of evolution slows, with the result that a bump occurs on the differential LF. As metallicity drops, the so-called RGB-bump occurs at progressively more evolved states (e.g. Fusi Pecci et al. 1990, Ferraro et al. 2000), and hence becomes more difficult to detect due to the increased statistical flucuations in star counts. Deep mixing in stars on the lower giant branch can affect the amplitude of the RGB-bump (Langer, Bolte, \& Sandquist 2000).

The integrated LF is a powerful tool for detecting the RGB-bump (e.g. Lee \& Carney 1999). A least squares fit was made to the integrated $K$ LF of each cluster between $K=13.5$ and $K=16$, and the RGB-bump was identified as the point after which the LF departs 
from this relation at the bright end. The RGB-bump was detected in four inner spheroid clusters using this method: NGC 6273 ( $K=13.2 \pm 0.1)$, NGC 6401 ( $K=13.2 \pm 0.1)$, NGC $6522(K=13.4 \pm 0.1)$, and NGC $6642(K=13.4 \pm 0.1)$, and the integrated LFs of these clusters are shown in Figure 12; the RGB-bump was not detected in the outer halo or the remaining inner spheroid clusters.

The RGB-bump will also produce a feature in differential LFs, although the signature is more difficult to detect given the lower signal-to-noise ratio. To check the RGB-bump brightnesses measured above, the differential LF for each cluster was compared with the power-laws that were fit to the entire RGB (§5). Significant departures from the power-law relations were detected in the differential $K$ LFs of NGC 6273, NGC 6401, NGC 6522, and NGC 6642 at the same brightnesses as found in Figure 12. The differential LFs of these clusters are shown in Figure 13, with the RGB-bump marked. The comparison in Figure 13 verifies the RGB-bump brightnesses measured from the integrated LFs.

The distance moduli of NGC 6273, NGC 6401, NGC 6522, and NGC 6642 were computed using the RGB-bump calibration given in the top panel of Figure 13 of Ferraro et al. (2000), and the results are listed in the second column of Table 4, where corrections for extinction have been made using the Rieke \& Lebofsky (1985) reddening law. Piotto et al. (1999) measured the $V$ brightness of the RGB-bump in NGC 6273, and found that $\mu_{0}=14.8$, which is in excellent agreement with the near-infrared RGB-bump distance modulus computed here.

Davidge (2000a) calculated distance moduli for NGC 6273, NGC 6522, and NGC 6642 using the brightnesses of the RGB-tip and the HB, and the results are listed in the third and fourth columns of Table 4. The mean difference between the distance moduli computed using the RGB-bump and RGB-tip is $0.3 \pm 0.4$, while the mean difference between the RGB-bump and HB results is $0.7 \pm 0.2$; in both cases the quoted uncertainties are the errors in the mean. The RGB-tip and HB distances given by Davidge (2000a) are based on the empirical Carney, Storm, \& Jones (1992) RR Lyrae calibration, while Ferraro et al. (2000) calibrate the RGB-bump using the theoretical ZAHB calibration given by Ferraro et al. (1999), which is consistent with Hipparcos sub-dwarf brightnesses. The Carney et al. (1992) and Ferraro et al. (1999) calibrations differ by roughly 0.2 mag at the metal-poor end, in the sense that the former predicts that RR Lyraes are intrinsically fainter. The application of a 0.2 mag correction to the RGB-tip and HB distance moduli in Table 4 brings these values into better agreement with the RGB-bump distances, although the spread between the various distance estimates for NGC 6642 is still very large.

Of the three features used to measure distances in Table 4, the RGB-tip is likely the most prone to systematic errors, due to (1) the rapid pace of evolution near the RGB-tip 
and (2) the tendency for bright giants to be depleted near the center of dynamically evolved clusters. Both of these sources of error will bias the measured RGB-tip brightness, causing it to be fainter than the actual value, with the result that distances will be overestimated. In fact, the RGB-tip and HB distances given by Davidge (2000a) for 19 metal-poor inner spheroid clusters differ by $\Delta \mu=0.43 \pm 0.13 \mathrm{mag}$, in the sense that the RGB-tip gives larger distances. The clusters that Trager et al. (1995) find are core-collapsed or have uncertain dynamical states tend to have the largest values of $\Delta \mu$, with an average $\Delta \mu=0.56 \pm 0.20$. For comparison, the clusters that are not core collapsed have an average $\Delta \mu=0.26 \pm 0.13$. Thus, this comparison suggests that dynamical evolution is the dominant source of systematic errors in the use of the RGB-tip as a distance indicator.

Distances to low Galactic latitude clusters computed from the HB are also subject to systematic effects introduced by contamination from the field HB component, which will bias distances towards that of the peak in the bulge stellar density distribution along the sight line. The brightness of the HB also varies by a few tenths of a magnitude among clusters of the same metallicity, likely due to age (e.g. Lee \& Carney 1999). The RGB-bump offers a means of measuring distances to metal-poor bulge clusters that should be less susceptible to systematic errors. In particular, the RGB-bump occurs in a portion of the CMD where bulge giants can be identified using CO indices (Davidge 2000a), so that field stars can be removed from heavily contaminated cluster fields. Models predict that the

brightness of the RGB-bump is only slightly sensitive to age, with $\frac{\Delta M_{V}}{\Delta t_{G y r}}=0.025 \mathrm{mag} \mathrm{Gyr}^{-1}$ (Ferraro et al. 1999). However, the RGB-bump is only detected in the most metal-rich clusters in the current sample, and data covering a significantly larger area will be required to detect the RGB-bump in the remaining, more metal-poor, clusters.

\section{DISCUSSION}

$J, H$, and $K$ images obtained with the CFHT AOB have been used to study the near-infrared photometric properties of evolved stars in eight metal-poor inner spheroid clusters. The CMDs show well-defined sequences, and artificial star experiments indicate that the scatter in these data is dominated by random errors. These data thus provide further evidence that photometric measurements with uncertainties of a few percent can be obtained of stars within 10 - 15 arcsec of the guide source in AO-compensated images. In $\S 7.1$ the results from the current work are discussed on a cluster-by-cluster basis, while in $\S 7.2$ the relative numbers of metal-poor and metal-rich clusters are used to probe the early evolution of the inner spheroid. 


\subsection{Comparisons with Previous Work}

Piotto et al. (1999) use $B$ and $V$ WFPC2 images to investigate the stellar content of NGC 6273, and point out that this cluster is in some respects similar to $\omega$ Cen. Based on the width of the $(V, B-V)$ CMD, Piotto et al. suggest that an abundance dispersion may be present, although differential reddening on small angular scales likely also contributes to smearing of the giant branch. The width of the NGC 6273 giant branch on the $(K, J-K)$ CMD in Figure 6 is similar to that of the other clusters, while the star-to-star distribution of $\mathrm{CO}$ indices is not markedly different from that of other metal-poor clusters (Davidge 2000a). Consequently, near-infrared observations do not support the presence of a significant metallicity dispersion near the center of NGC 6273, suggesting that the smearing of the giant branch at visible wavelengths is a consequence of differential reddening.

Rutledge, Hesser, \& Stetson (1997) measured the strength of the near-infrared Ca triplet in the integrated spectrum of NGC 6273 and concluded that $[\mathrm{Fe} / \mathrm{H}]=-1.80 \pm 0.03$ using the Zinn \& West (1984) calibration, and $-1.53 \pm 0.05$ using the Carretta \& Gratton (1997) calibration. The metallicity of NGC 6273 derived from the slope of the giant branch with the current data is consistent with the latter result.

As the most metal-poor cluster yet discovered in the inner spheroid, NGC 6287 has the potential of providing important clues into the early evolution of the inner Galaxy. Stetson \& West (1994) compared the $(V, B-V)$ CMD of NGC 6287 with that of the metal-poor halo cluster M15, and found that the HB brightnesses of these clusters differ by a few tenths of a mag, in the sense that NGC 6287 is the fainter of the two. The giant branch of NGC 6287 is steeper than that of other metal-poor clusters, such as M92, on the $(K, J-K)$ CMD (Davidge 1998; Davidge \& Courteau 1999); in fact, the CMDs in Figures 6 and 8 show a tendency for cluster giant branches to become shallower when $K<12$, but for NGC 6287 the giant branch slope appears not to change near the RGB-tip.

Given the extreme metal-poor nature of NGC 6287, it is important to determine if this cluster formed as part of the inner spheroid. The compact nature of NGC 6287 indicates that it is likely not an interloper from the outer halo (van den Bergh, Morbey, \& Pazder 1991). However, compactness is not an unambigous criterion for membership in the inner spheroid, since the globular clusters in the Fornax dwarf spheroidal galaxy are also compact (van den Bergh 1994). Consequently, the possibility that NGC 6287 may not have formed as part of the inner spheroid cluster system, but may have been stripped from a dwarf galaxy that was accreted by the Milky-Way (e.g. van den Bergh 2000), can not at this time be ruled out.

Janes \& Heasley (1991) used CCD data to investigate the $(V, B-V)$ CMDs of NGC 
6293 and NGC 6333, and found that the giant branches of both clusters are well matched by that of M92, although Ferraro et al. (1999) note that the RGB locus of NGC 6333 defined by the Janes \& Heasley (1991) data crosses the giant branches of other clusters, possibly indicating uncertainties in the photometric calibration. The slopes of the giant branches of NGC 6293 and NGC 6333 on the $(K, J-K)$ CMDs are consistent with a very low metallicity. Janes \& Heasley (1991) also found that the upper giant branch of NGC 6293 is poorly populated, possibly due to dynamical evolution. The power-law exponent of the RGB K LF of NGC 6293 is similar to that of the other core-collapsed clusters in this sample, which as a group tend to have flatter LFs than non-collapsed clusters.

NGC 6355 is the least studied cluster in the current sample, and the only published CMD is that presented by Davidge (2000a), who found that $[\mathrm{M} / \mathrm{H}]=-1.6$ based on comparisons with theoretical isochrones. The slope of the RGB on the $(K, J-K)$ CMD in Figure 6 confirms that NGC 6355 is very metal-poor, and hence is a potentially important target for studies of the age of the inner spheroid.

Barbuy et al. (1999) obtained $V$ and $I$ images of NGC 6401, and their CMD of stars between 1.3 and 2.0 arcmin from the cluster center shows a red HB and a curved upper giant branch similar to that in 47 Tuc. However, the slope of the giant branch on the near-infrared CMD published by Minniti et al. (1995) is comparable to that of M92, suggesting that NGC 6401 is very metal-poor. Minniti (1995b) obtained visible spectra of bright giants in NGC 6401, which radial velocity measurements suggest are cluster members, and concluded that $[\mathrm{Fe} / \mathrm{H}]=-1.1 \pm 0.2$. The slope of the NGC $6401 \mathrm{RGB}$ on the $(K, J-K)$ CMD in Figure 6 indicates that $[\mathrm{Fe} / \mathrm{H}]=-1.5$. The current data also indicate that the HB of this cluster is uniformly populated over a range of $J-K$ colors, as expected if the cluster is old and moderately metal-poor. Thus, it appears that NGC 6401 is metal-poor, and not metal-rich.

Because it is located in BW, NGC 6522 is the most extensively studied cluster in the inner spheroid. The CMD of NGC 6522 at visible wavelengths shows a giant branch with a color and slope characteristic of low metallicities (Terndrup \& Walker 1994; Barbuy, Ortolani, \& Bica 1994). Rutledge et al. (1997) use the depth of Ca II absorption in the intregrated cluster spectrum to assign $[\mathrm{Fe} / \mathrm{H}]=-1.50 \pm 0.05$ on the Zinn \& West (1984) metallicity scale, and $[\mathrm{Fe} / \mathrm{H}]=-1.21 \pm 0.04$ on the Carretta \& Gratton (1997) scale. The metallicity derived from the giant branch slope in the present study is consistent with the former measurement.

Shara et al. (1998) used WFPC2 data to examine the $(B, U-B)$ CMD of stars near the center of NGC 6522, and found that the upper giant branch population is depleted within 9 arcsec of the cluster center. Rich et al. (1998) suggest that NGC 6522 belongs to a 
class of dynamically evolved clusters, including HP 1, NGC 6540, and NGC 6558, that are unique to the inner spheroid. The current data indicate that the $K$ LF of giants in NGC 6522 has a power-law exponent that is consistent with other core-collapsed inner spheroid clusters.

$(K, J-K)$ CMDs of bright giants in NGC 6642 have been presented by Davidge (2000a) and Minniti et al. (1995). The latter found a relatively shallow giant branch, suggesting that NGC 6642 is moderately metal-rich, while the former found that $[\mathrm{M} / \mathrm{H}]=$ -1.9. Minniti (1995b) concluded that $[\mathrm{Fe} / \mathrm{H}]=-1.4 \pm 0.2$ based on the strengths of $\mathrm{Mg}$ and Fe indices in the visible spectra of cluster giants, and this is consistent with the metallicity measured from the slope of the giant branch in the current work. Thus, NGC 6642 is a moderately metal-poor cluster.

\subsection{Globular Clusters and the Formation of the Inner Galaxy}

Stars with $[\mathrm{Fe} / \mathrm{H}]<-1$ account for only a small fraction of the total field population in BW (e.g. McWilliam \& Rich 1994). However, the June 1999 compilation of Harris (1996) indicates that the ratio of clusters within $3 \mathrm{kpc}$ of the $\mathrm{GC}$ with $[\mathrm{Fe} / \mathrm{H}]>-1$ to those with $[\mathrm{Fe} / \mathrm{H}]<-1$ is $21 / 22=0.95$. Thus, when measured with respect to field stars of similar metallicity, the frequency of metal-poor clusters in the inner Galaxy is much higher than that of metal-rich clusters.

Various models (e.g. Fall \& Rees 1985; Harris \& Pudritz 1994, and McLaughlin \& Pudritz 1996) argue that globular cluster formation requires conditions that occur during the initial phases of protogalactic collapse. Another prediction is that globular clusters form before the field population (e.g. Fall \& Rees 1985), and this can explain why globular clusters in external galaxies tend to be more metal-poor than the underlying starlight (Forbes et al. 1996; Da Costa \& Mould 1988). These formation models, when considered with observation evidence, suggest that globular clusters are not good direct tracers of the overall star-forming histories of galaxies (van den Bergh 1998), and the difference in the relative frequencies of metal-poor and metal-rich clusters supports this notion. However, the relative frequencies of metal-poor and metal-rich clusters may still provide indirect clues into the early evolution of the inner spheroid.

Harris, Harris, \& McLaughlin (1998) investigated the specific globular cluster frequencies of brightest cluster galaxies, and suggested that the suppression of star formation after an initially large burst of activity, during which time globular clusters formed and star-forming material was ejected by stellar and supernovae-driven winds, 
could be responsible for the high specific cluster frequencies among these systems. When considered in the context of the Harris et al. (1998) scenario, the relative frequencies of metal-poor and metal-rich clusters in the inner Galaxy could be interpreted as being due to an early large spike in the star formation rate, at which time the metal-poor clusters formed. The formation of metal-poor field stars was stopped early-on by the ejection of gas, but star-forming activity was subsequently rejuvenated when chemically-enriched gas fell back into the central Galaxy, at which time the metal-rich clusters and field stars formed.

Wyse \& Gilmore (1992) discuss the possible sources of gas from which the bulge formed, and argue that this material could not have come from the halo. If the metal-poor globular clusters in the inner spheroid formed during an initial spike in the star-formation rate, as suggested above, then some bulge stars may have formed from the metal-enriched gas that was ejected by SNII following this event, and so should have non-solar abundances of elements such as oxygen (see also Wyse \& Gilmore 1992). McWilliam \& Rich (1994) find that at least some chemical elements in bulge giants are enriched with respect to solar.

The scenario described above predicts that metal-poor clusters are older than metal-rich clusters, and the existence of such an age-metallicity relation remains a matter of controversy. While some studies find at least marginal evidence for such a relation (e.g. Chaboyer, Demarque, \& Sarajedini 1996; Richer et al. 1996; Salaris \& Weiss 1998; Rosenberg et al. 1999), others conclude that the metal-rich and metal-poor clusters are coeval (e.g. Ortolani et al. 1995; Buonanno et al. 1998). The various sources of uncertainty that frustrate efforts to compare the ages of clusters spanning a broad range of metallicities are discussed by Rosenberg et al. (1999).

The chemical composition of cluster stars provides broad constraints on the time delay between the formation of the metal-poor and metal-rich clusters, and the existing data, while not conclusive, suggest that any age difference may prove difficult to measure. If the metal-poor and metal-rich clusters have similar $\alpha$ element abundances, as suggested by Carney (1996), then the clusters formed from material that was enriched on a timescale that is shorter than the onset of SN I ( $\leq 1 \mathrm{Gyr}$ ), and age differences of this size would be challenging to detect. However, if the metal-rich clusters show a trend of decreasing $\alpha$ enrichment towards higher metallicities, as is the case among field stars and may be the case among globular clusters (Ferraro et al. 1999), then the cluster system formed from material that was enriched over a longer time span.

The mass of gas that was initially present in the inner Galaxy can be estimated knowing (1) the total mass of globular clusters, and (2) the globular cluster formation efficiency, $\epsilon$, which is the ratio of mass in globular clusters to that in stars and gas (McLaughlin 1999). As noted by McLaughlin (1999), $\epsilon$ is a more physical measure of cluster 
statistics than the traditional specific frequency, $\mathrm{S}_{N}$, as $\epsilon$ accounts for sources of mass that are not accounted for in $\mathrm{S}_{N}$, such as gas in extended halos. Harris et al. (1998) argue that $\epsilon$ is a constant that holds over a range of different environments.

There are 22 metal-poor and 21 metal-rich globular clusters with $\mathrm{R}_{G C} \leq 3 \mathrm{kpc}$ (Harris 1996) and, if each has a mass of $2 \times 10^{5} \mathrm{M}_{\odot}$ (McLaughlin 1999), then the total mass of these objects is:

$$
43 \times 2 \times 10^{5}=8.6 \times 10^{6} \mathrm{M}_{\odot}
$$

This is only a lower limit to the total mass of the early cluster system, since (1) globular clusters in the inner Galaxy are dissrupted by dynamical interactions, and (2) incompleteness in the present-day cluster population has not been accounted for, although the number of missed clusters is likely not large, and will not be considered further. Simulations conducted by Vesperini (1997) suggest that only a third of the current cluster population has survived in the central $3 \mathrm{kpc}$. Therefore, the total initial mass of clusters is:

$$
3 \times 8.6 \times 10^{6}=2.6 \times 10^{7} \mathrm{M}_{\odot}
$$

McLaughlin (1999) computed $\epsilon$ for three massive early-type galaxies, and found a mean value $\bar{\epsilon}=0.0027$ with only modest galaxy-to-galaxy scatter. If this value of $\epsilon$ is also assumed to hold for the inner spheroid cluster system, then the globular clusters formed from an initial supply of gas that had a mass:

$$
\frac{2.6 \times 10^{7}}{2.7 \times 10^{-3}}=10^{10} \mathrm{M}_{\odot}
$$

The mass of the bulge within $3 \mathrm{kpc}$ is roughly $10^{10} \mathrm{M}_{\odot}$ (Blanco \& Terndrup 1989), and this is in remarkable agreement with the gas mass computed above. It thus appears that during the earliest episodes of star formation in the inner Galaxy there was a large reservoir of material from which a significant fraction of the current bulge population could have been assembled. Of course, star formation is an inefficient process, and only some fraction of the available gas will be turned into stars. Adopting the relatively high efficiency of $10 \%$ that is required to produce bound clusters (Elmegreen \& Clemens 1985), then only $10^{9} \mathrm{M}_{\odot}$ of stars would be formed during any large-scale star-forming episode in the inner spheroid; however, the cumulative efficiency due to subsequent star-forming episodes will be much higher if gas is re-cycled. These admittedly simple calculations indicate that it may not be necessary to invoke a separate pre-enriched body of gas to form a large fraction of the bulge; rather, the early enrichment of this material may have occured in situ.

Sincere thanks are extended to Sidney van den Bergh for commenting on an earlier version of this paper, and to Peter Stetson for discussions concerning the ages of globular 
clusters. An anonymous referee also provided comments that greatly improved the paper. 


\section{REFERENCES}

Barbuy, B., Ortolani, S., \& Bica, E. 1994, A\&Ap, 285, 871

Barbuy, B., Ortolani, S., Bica, E., \& Desidera, S. 1999, A\&Ap, 348, 783

Bertelli, G., Bressan, A., Chiosi, C., Fagotto, F., \& Nasi, E. 1994, A\&AS, 106, 275

Blanco, V. M., \& Terndrup, D. M. 1989, AJ, 98, 843

Buonanno, R., Corsi, C., Bellazzini, M., Ferraro, F. R., \& Fusi Pecci, F. 1997, AJ, 113, 706

Buonanno, R., Corsi, C. E., Pulone, L., Fusi Pecci, F., \& Bellazzini, M. 1998, A\&A, 333, 505

Burgarella, D., \& Buat, V. 1996, A\&A, 313, 129

Burkert, A., \& Smith, G. H. 1997, ApJ, 474, L15

Carney, B. W. 1996, PASP, 108, 900

Carney, B. W., Storm, J., \& Jones, R. V. 1992, ApJ, 386, 663

Carretta, E., \& Gratton, R. G. 1997, A\&AS, 121, 95

Casali, M., \& Hawarden, T. 1992, JCMT-UKIRT Newsletter, 4, 33

Chaboyer, B., Demarque, P., Sarajedini, A. 1996, ApJ, 459, 558

Côté, P. 1999, AJ, 118, 406

Da Costa, G. S., \& Mould, J. R. 1988, ApJ, 334, 159

Davidge, T. J. 1991, AJ, 103, 1259

Davidge, T. J. 1995, AJ, 110, 1177

Davidge, T. J. 1998, AJ, 116, 1744

Davidge, T. J. 2000a, AJ, 120, 1853

Davidge, T. J. 2000b, ApJS, 126, 105

Davidge, T. J., \& Courteau, S. 1999, AJ, 117, 1297

Djorgovski, S., Piotto, G., Phinney, E. S., \& Chernoff, D. F. 1991, ApJ, 372, L41

Djorgovski, G., \& King, I. R. 1986, ApJ, 305, L61

Djorgovski, S., \& Piotto, G. 1993, in Structure and Dynamics of Globular Clusters, ASP Conference Series \# 50, ed. S. G. Djorgovski \& G. Meylan, pp. 203

Elmegreen, B. G., \& Clemens, C. 1985, ApJ, 294, 523

Fall, S. M., \& Rees, M. J. 1985, ApJ, 298, 18 
Ferraro, F. R., Messineo, M., Fusi pecci, F., De Palo, M. A., Straniero, O., Chieffi, A., \& Limongi, M. 1999, AJ, 118, 1738

Ferraro, F. R., Montegriffo, P., Origlia, L., \& Fusi Pecci, F. 2000, AJ, 119, 1282

Forbes, D. A., Franx, M., Illingworth, G. D., \& Carollo, C. M. 1996, ApJ, 467, 126

Fusi Pecci, F., Ferraro, F. R., Crocker, D. A., Rood, R. T., \& Buonanno, R. 1990, A\&A, 238, 95

Harris, W. E. 1996, AJ, 112, 1487

Harris, W. E., \& Pudritz, R. E. 1994, ApJ, 429, 177

Harris, W. E., Harris, G. L. H., \& McLaughlin, D. E. 1998, AJ, 115, 1801

Heitsch, F., \& Richtler, T. 1999, A\&A, 347, 455

Iben, I. 1968, Nature, 220, 143

Ivanov, V. D., Borissova, J., Alonso-Herrero, A., \& Russeva, T. 2000, AJ, 119, 2274

Janes, K. A., \& Heasley, J. N. 1991, AJ, 101, 2097

Kuchinsky, L. E., Frogel, J. A., Terndrup, D. M., \& Persson, S. E. 1995, AJ, 109, 1131

Langer, G. E., Bolte, M., \& Sandquist, E. 2000, ApJ, 529, 936

Larson, R. B. 1990, PASP, 102, 709

Lee, J-W, \& Carney, B. W. 1999, AJ, 118, 1373

Lee, Y.-W., Demarque, P., \& Zinn, R. J. 1994, ApJ, 423, 248

McClure, R. D., Arnaud, J., Fletcher, J. M., Nieto, J-L, \& Racine, R. 1991, PASP, 103, 570

McLaughlin, D. E. 1999, AJ, 117, 2398

McLaughlin, D. E., \& Pudritz, R. E. 1996, ApJ, 457, 578

McWilliam, A., \& Rich, R. M. 1994, ApJS, 91, 749

Minniti, D. 1995a, AJ, 109, 1663

Minniti, D. 1995b, A\&A, 303, 468

Minniti, D. 1996, ApJ, 459, 175

Minniti, D., Olszewski, E. W., \& Rieke, G. H. 1995, AJ, 110, 1686

Ortolani, S., Barbuy, B., Bica, E., Renzini, A., Marconi, G., Gilmozzi, R. 1999, A\&A, 350, 840

Ortolani, S., Renzini, A., Gilmozzi, R., Marconi, G., Barbuy, B., Bica, E., \& Rich, R. M. 1995, Nature, 377, 701 
Piotto, G. et al. 1999, AJ, 118, 1727

Reid, M. 1993, ARAA, 31, 345

Rich, R. M., Ortolani, S., Bica, E., \& Barbuy, B. 1998, AJ, 116, 1295

Richer, H. B. et al. 1996, ApJ, 463, 602

Rieke, G. H., \& Lebofsky, M. J. 1985, ApJ, 288, 618

Rigaut, F. et al. 1998, PASP, 110, 152

Rosenberg, A., Saviane, I., Piotto, G., \& Aparicio, A. 1999, AJ, 118, 2306

Rutledge, G. A., Hesser, J. E., \& Stetson, P. B. 1997, PASP, 109, 907

Salaris, M., \& Weiss, A. 1997, A\&A, 327, 107

Salaris, M., \& Weiss, A. 1998, A\&A, 335, 943

Searle, L., \& Zinn, R. 1978, ApJ, 225, 357

Shara, M. M., Drissen, L., Rich, R. M., Paresce, F., King, I. R., \& Meylan, G. 1998, ApJ, 495,796

Stetson, P. B. 1987, PASP, 99, 191

Stetson, P. B., \& Harris, W. E. 1988, AJ, 96, 909

Stetson, P. B., \& West, M. J. 1994, PASP, 106, 726

Terndrup, D. M., \& Walker, A. R. 1994, AJ, 107, 1786

Trager, S. C., King, I. R., \& Djorgovski, S. 1995, AJ, 109, 218

van den Bergh, S. 1994, AJ, 108, 2145

van den Bergh, S. 1998, ApJ, 507, L39

van den Bergh, S. 2000, ApJ, 530, 777

van den Bergh, S., Morbey, C., \& Pazder, J. 1991, ApJ, 375, 594

Vesperini, E. 1997, MNRAS, 287, 915

Wyse, R. F. G., \& Gilmore, G. 1992, AJ, 104, 144

Zinn, R., \& West, M. J. 1984, ApJS, 55, 45 


\begin{tabular}{lcccc}
\hline \hline Cluster & {$[\mathrm{Fe} / \mathrm{H}]$} & $\mathrm{E}(\mathrm{B}-\mathrm{V})$ & $\begin{array}{c}\mathrm{R}_{G C} \\
(\mathrm{kpc})\end{array}$ & $\begin{array}{c}\text { Core-Collapsed? } \\
(\text { Yes/No })\end{array}$ \\
\hline NGC 6273 & -1.68 & 0.37 & 1.6 & $\mathrm{~N}$ \\
NGC 6287 & -2.05 & 0.60 & 1.7 & $\mathrm{~N}$ \\
NGC 6293 & -1.92 & 0.41 & 1.4 & $\mathrm{Y}$ \\
NGC 6333 & -1.72 & 0.38 & 1.7 & $\mathrm{~N}$ \\
NGC 6355 & -1.50 & 0.75 & 1.0 & $\mathrm{Y}$ \\
NGC 6401 & -1.12 & 0.85 & 0.8 & $\mathrm{~N}$ \\
NGC 6522 & -1.44 & 0.48 & 0.6 & $\mathrm{Y}$ \\
NGC 6642 & -1.35 & 0.41 & 1.6 & $\mathrm{Y} ?$ \\
& & & & \\
NGC 288 & -1.24 & 0.03 & 11.6 & $\mathrm{~N}$ \\
M13 & -1.54 & 0.02 & 8.7 & $\mathrm{~N}$ \\
M92 & -2.29 & 0.02 & 9.6 & $\mathrm{~N}$ \\
\hline
\end{tabular}

Table 1: CLUSTER PROPERTIES 


\begin{tabular}{lcc}
\hline \hline Cluster & $\frac{\Delta(J-K)}{\Delta(K)}$ & {$[\mathrm{M} / \mathrm{H}]_{\text {Ferraroetal. }}$} \\
\hline NGC 6273 & $-0.069 \pm 0.002$ & $-1.4 \pm 0.2$ \\
NGC 6287 & $-0.031 \pm 0.004$ & $-2.3 \pm 0.2$ \\
NGC 6293 & $-0.033 \pm 0.004$ & $-2.2 \pm 0.2$ \\
NGC 6333 & $-0.037 \pm 0.003$ & $-2.1 \pm 0.2$ \\
NGC 6355 & $-0.041 \pm 0.005$ & $-2.0 \pm 0.2$ \\
NGC 6401 & $-0.065 \pm 0.003$ & $-1.5 \pm 0.2$ \\
NGC 6522 & $-0.063 \pm 0.004$ & $-1.5 \pm 0.2$ \\
NGC 6642 & $-0.055 \pm 0.005$ & $-1.7 \pm 0.2$ \\
& & \\
NGC 288 & $-0.054 \pm 0.006$ & - \\
M13 & $-0.049 \pm 0.004$ & $-1.8 \pm 0.2$ \\
M92 & $-0.035 \pm 0.006$ & $-2.2 \pm 0.2$ \\
\hline
\end{tabular}

Table 2: GIANT BRANCH SLOPES AND METALLICITIES 


\begin{tabular}{ccc}
\hline \hline Cluster & $x$ & $x_{\text {Davidge 2000a }}$ \\
\hline NGC 6273 & $0.34 \pm 0.03$ & $0.23 \pm 0.02$ \\
NGC 6287 & $0.22 \pm 0.03$ & $0.38 \pm 0.02$ \\
NGC 6293 & $0.28 \pm 0.02$ & $0.25 \pm 0.07$ \\
NGC 6333 & $0.40 \pm 0.02$ & $0.20 \pm 0.08$ \\
NGC 6355 & $0.26 \pm 0.02$ & $0.19 \pm 0.07$ \\
NGC 6401 & $0.41 \pm 0.03$ & - \\
NGC 6522 & $0.27 \pm 0.04$ & $0.31 \pm 0.07$ \\
NGC 6642 & $0.31 \pm 0.04$ & $0.17 \pm 0.03$ \\
& & \\
NGC 288 & $0.24 \pm 0.06$ & $0.22 \pm 0.03$ \\
M13 & $0.34 \pm 0.05$ & - \\
M92 & $0.29 \pm 0.02$ & - \\
\hline
\end{tabular}

Table 3: LUMINOSITY FUNCTION EXPONENTS 


\begin{tabular}{cccc}
\hline \hline Cluster & $\mu_{0}^{\text {RGB-bump }}$ & $\mu_{0}^{\text {RGB-tip }}$ & $\mu_{0}^{H B}$ \\
\hline NGC 6273 & 14.8 & 14.1 & 14.5 \\
NGC 6401 & 14.6 & - & - \\
NGC 6522 & 15.1 & 14.4 & 14.1 \\
NGC 6642 & 15.2 & 15.6 & 14.3 \\
\hline
\end{tabular}

Table 4: CLUSTER DISTANCES 


\section{FIGURE CAPTIONS}

Fig. 1.- The final deep $K$ images of NGC 6273 (upper left hand corner), NGC 6287 (upper right hand corner), NGC 6293 (lower left hand corner), and NGC 6333 (lower right hand corner). Each image is $34 \times 34$ arcsec, with North at the top, and East to the left. The AO reference stars are located near the field centers.

Fig. 2.- The final deep $K$ images of NGC 6355 (upper left hand corner), NGC 6401 (upper right hand corner), NGC 6522 (lower left hand corner), and NGC 6642 (lower right hand corner). Each image is $34 \times 34$ arcsec, with North at the top, and East to the left. The AO reference stars are located near the field centers.

Fig. 3.- $K$ images of a field near the center of NGC 6287, obtained during March 1998 (left hand panel) and September 1998 (right hand panel). The guide star for the March 1998 observations is marked with an 'M', while the guide star for the September 1998 observations is marked with an ' $\mathrm{S}$ '. The stars used to investigate the effects of anisoplanicity on the photometric measurements are indicated with circles.

Fig. 4. - The top two panels show the histogram distributions of the difference in $K$ brightness measured from the March 1998 and September 1998 datasets for the stars marked in Figure 3. The angular intervals refer to distances from star ' $\mathrm{S}$ ' in Figure 3. The means of the two distributions differ at only the $1.6 \sigma$ significance level. The lower panel shows the relation between $K$ and $\Delta K$; crosses are stars within 7 arcsec of star ' $\mathrm{S}$ ', while the solid squares are stars located between 7 and 14 arcsec of this star. Note that the extreme values in $\Delta K$ occur for stars that are near the faint limit of the September 1998 dataset.

Fig. 5.- The $(K, H-K)$ CMDs of the inner spheroid clusters. Note the modest scatter at intermediate brightnesses; the standard deviation in $H-K$ for stars between $K=12$ and 14 is typically \pm 0.02 to $\pm 0.03 \mathrm{mag}$, which is consistent with that predicted from artificial star experiments. The NGC 6287 data are from Davidge \& Courteau (1999).

Fig. 6. - The $(K, J-K)$ CMDs of the inner spheroid clusters. The CMDs terminate near the MSTO, and the HB can be seen to the left of the RGB between $\mathrm{K}=13$ and 16 . The NGC 6287 data are from Davidge \& Courteau (1999).

Fig. 7. - The $(K, H-K)$ CMDs of the outer halo clusters. The M13 and M92 data are from Davidge \& Courteau (1999).

Fig. 8.- The $(K, J-K)$ CMDs of the outer halo clusters. The M13 and M92 data are from Davidge \& Courteau (1999). 
Fig. 9.- Composite $(K, J-K)$ CMDs of the inner spheroid clusters grouped according to metallicity. The left hand panel shows the CMDs of NGC 6273, NGC 6401, NGC 6522, and NGC 6642, while the central panel shows the CMDs of NGC 6401 and NGC 6642, which have the best-defined HB sequences in the moderately metal-poor group. The right hand panel shows the CMDs of NGC 6287, NGC 6293, NGC 6333, and NGC 6355. Cluster-tocluster differences in reddening have been removed using the procedure described in the text. $\Delta(\mathrm{K})$ and $\Delta(\mathrm{J}-\mathrm{K})$ are measured from the shifted giant branches at $\mathrm{K}=14$. There are clear differences between the HBs of the $[\mathrm{Fe} / \mathrm{H}]=-1.5$ and $[\mathrm{Fe} / \mathrm{H}]=-2$ groups.

Fig. 10.- The K LFs of giant branch stars in inner spheroid clusters. The LFs were constructed from the CMDs, and HB stars have been removed. The solid lines are the raw LFs, while the dashed lines are the LFs corrected for incompleteness. $\mathrm{n}_{05}$ is the number of stars per 0.5 magnitude interval per square arcsec. The dotted lines are power-laws, the exponents for which are listed in the second column of Table 3, that were fit to the completeness-corrected LFs between $K=12$ and $K=16$.

Fig. 11.- Same as Figure 10, but for the outer halo clusters.

Fig. 12.- The integrated $K$ LFs of bright giant branch stars in NGC 6273, NGC 6401, NGC 6522, and NGC 6642, where secure RGB-bump detections have been made. $\Sigma \mathrm{N}_{02}$ is the cumulative number of stars per 0.2 magnitude interval, and the error bars show the uncertainties introduced by counting statistics. The arrows mark the location of the RGBbump, which is defined as the point after which the star counts depart from the relation predicted by stars between $K=13.5$ and 15 , which is shown as a solid line.

Fig. 13. - The differential $K$ LFs of bright giant branch stars in those clusters for which the RGB-bump has been identified. $\mathrm{N}_{02}$ is the number of stars per 0.2 magnitude interval, and the error bars show the uncertainties introduced by counting statistics. The dashed line shows a least squares fit to the giant branch LF between $K=12$ and 16 for each cluster, and the arrows mark the location of the RGB-bump predicted from the integrated LFs in Figure 12. In all four clusters the star counts at the expected RGB-bump brightness exceed, at the $2-\sigma$ or higher significance level, those expected from the least squares fit, thus confirming the detection of this feature. 


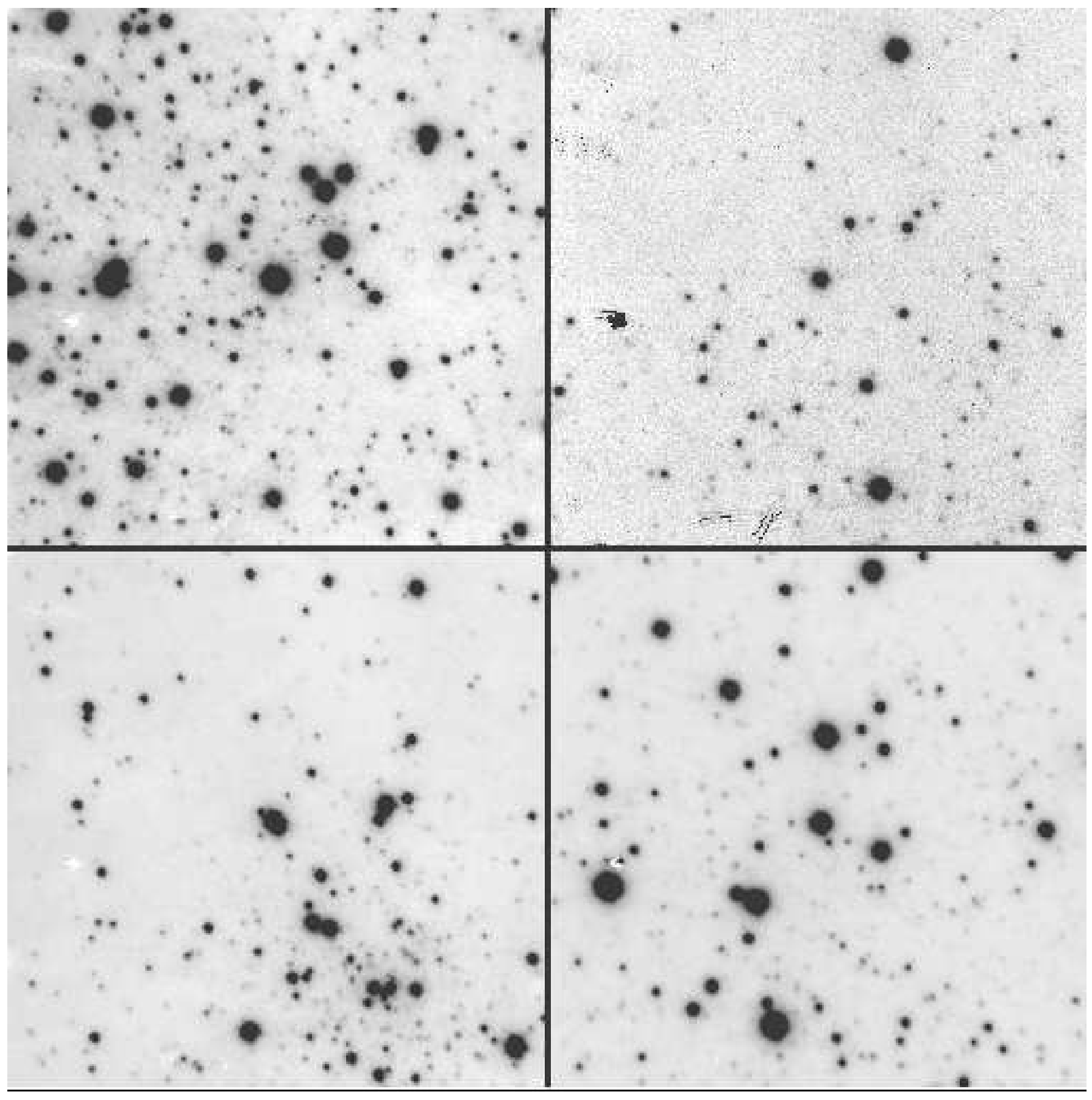




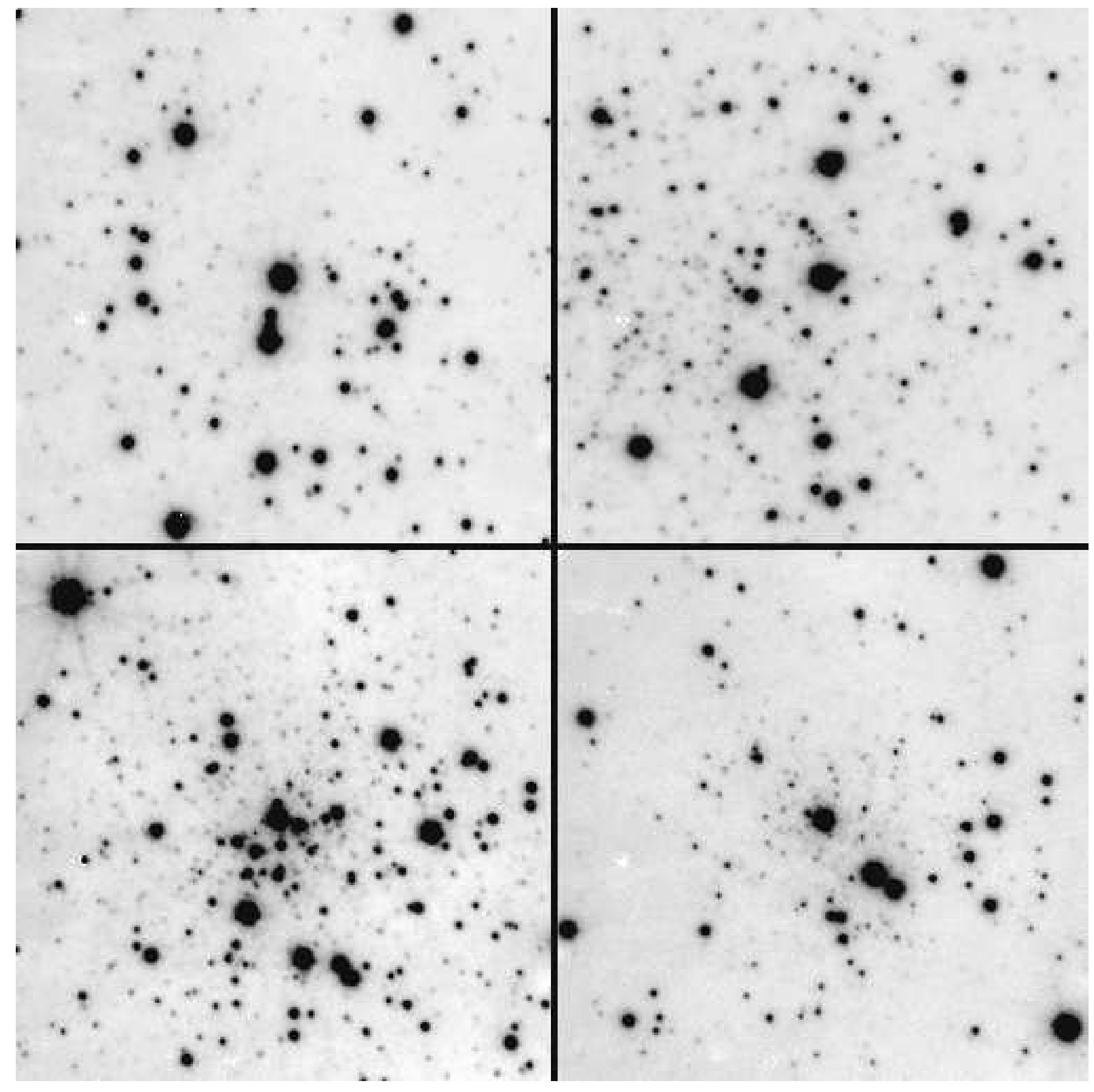




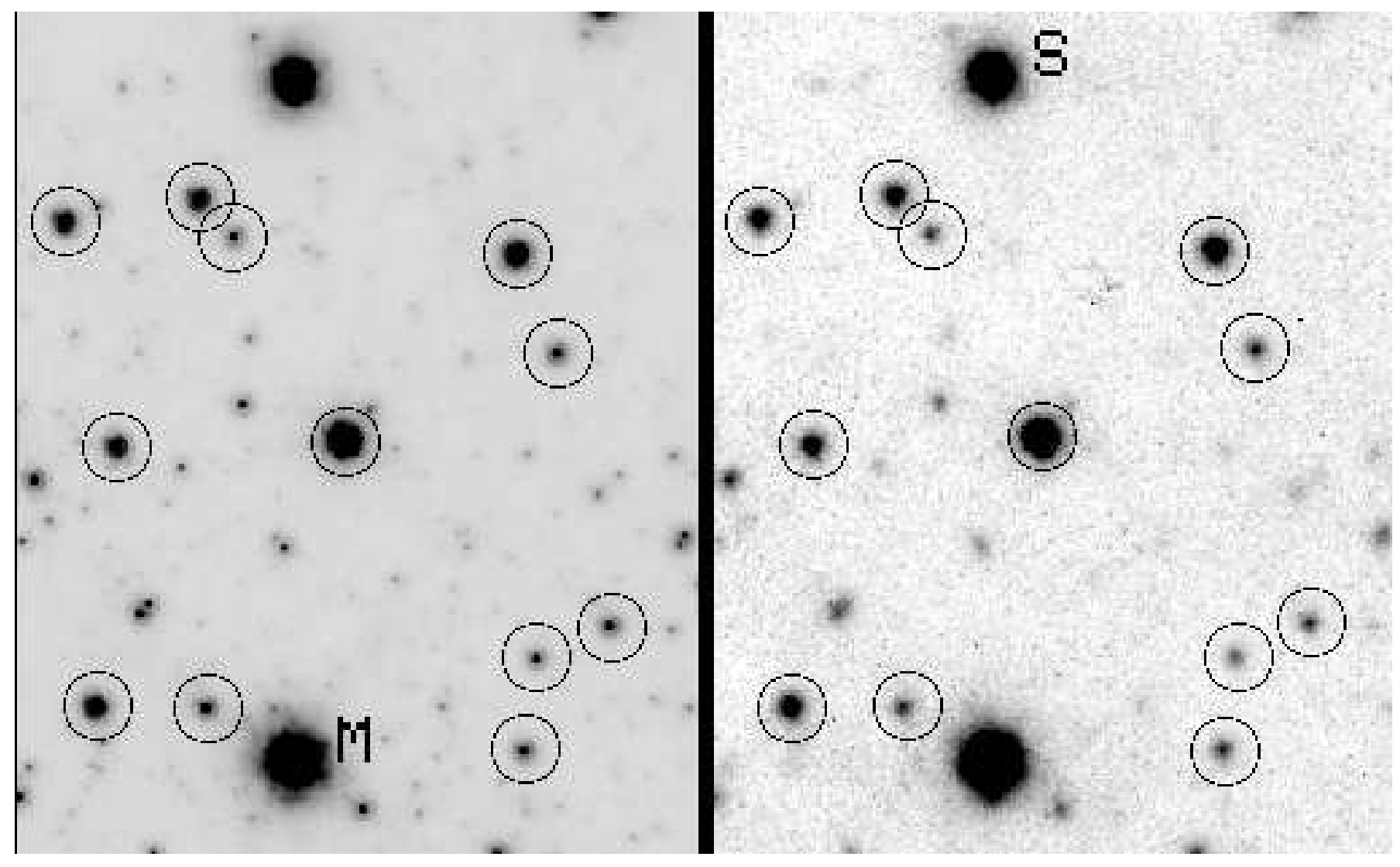




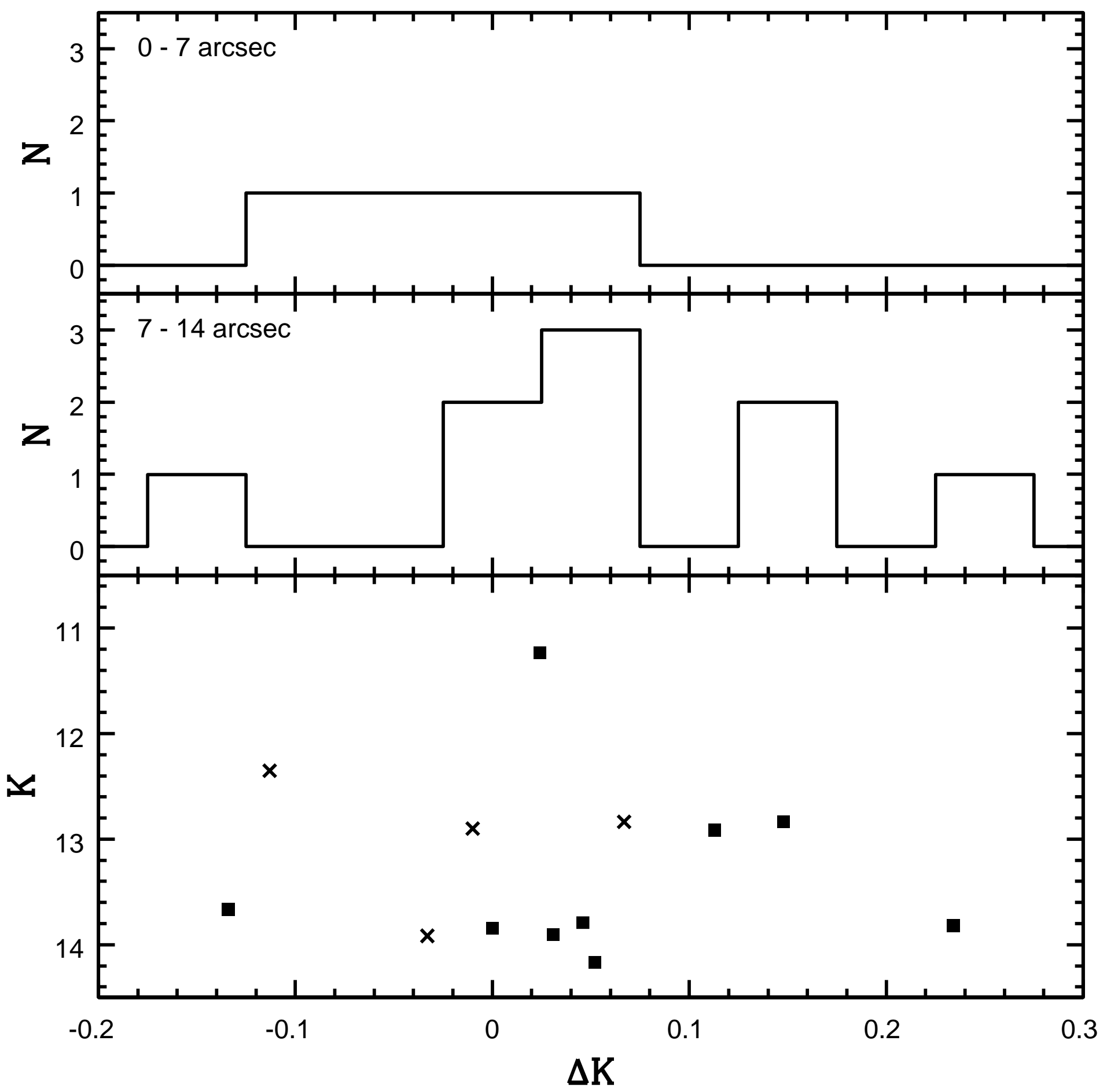




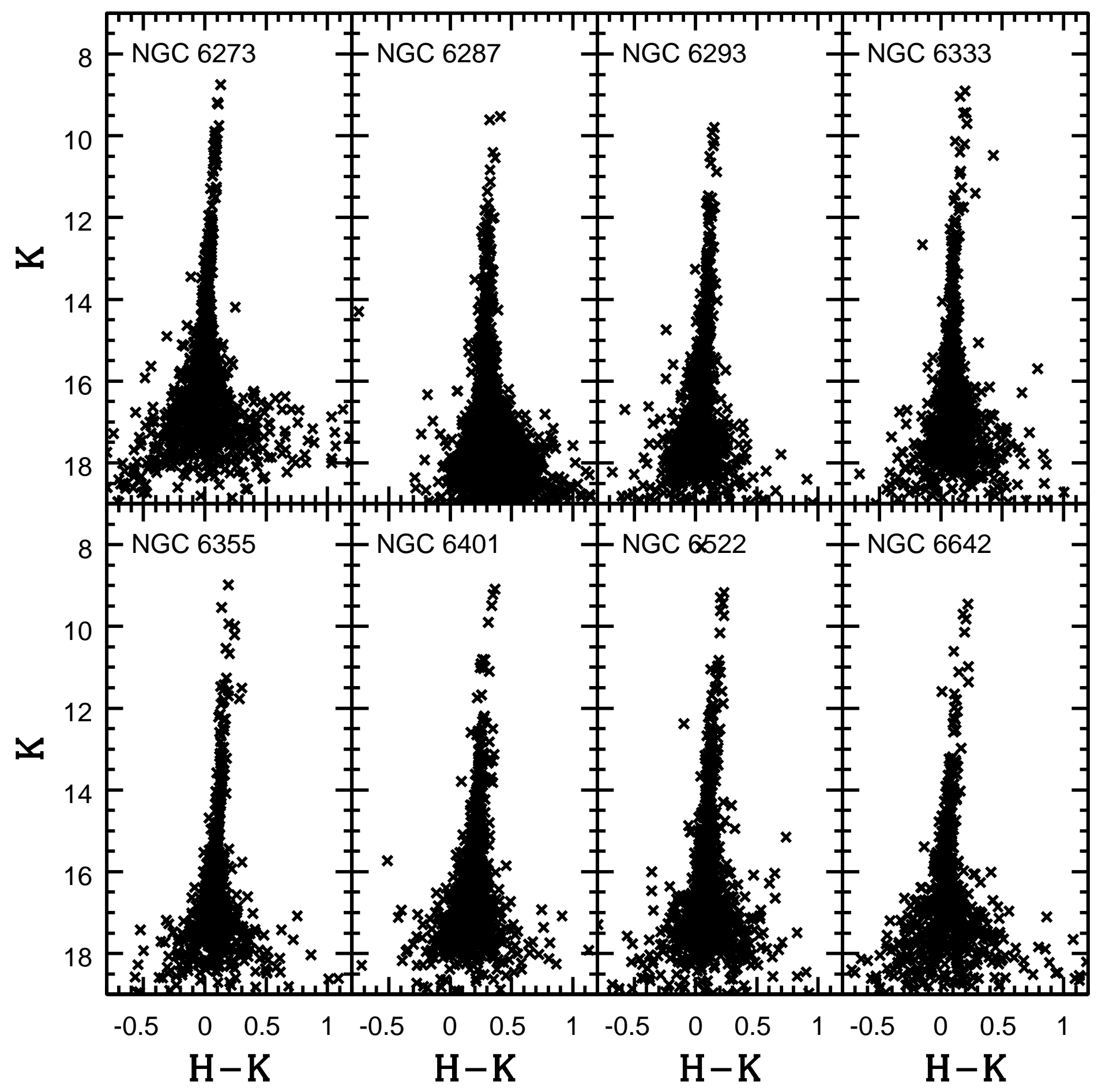




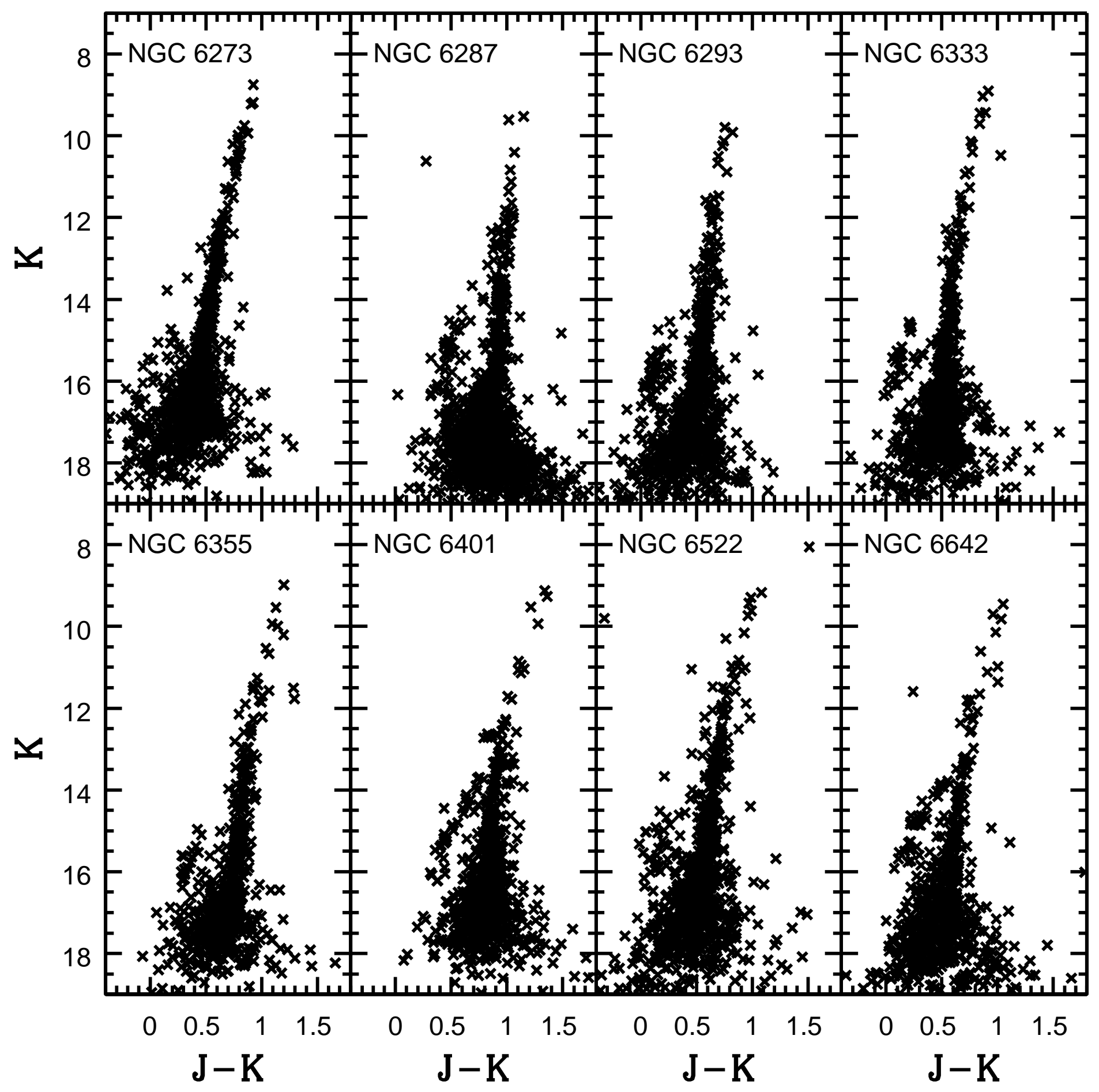




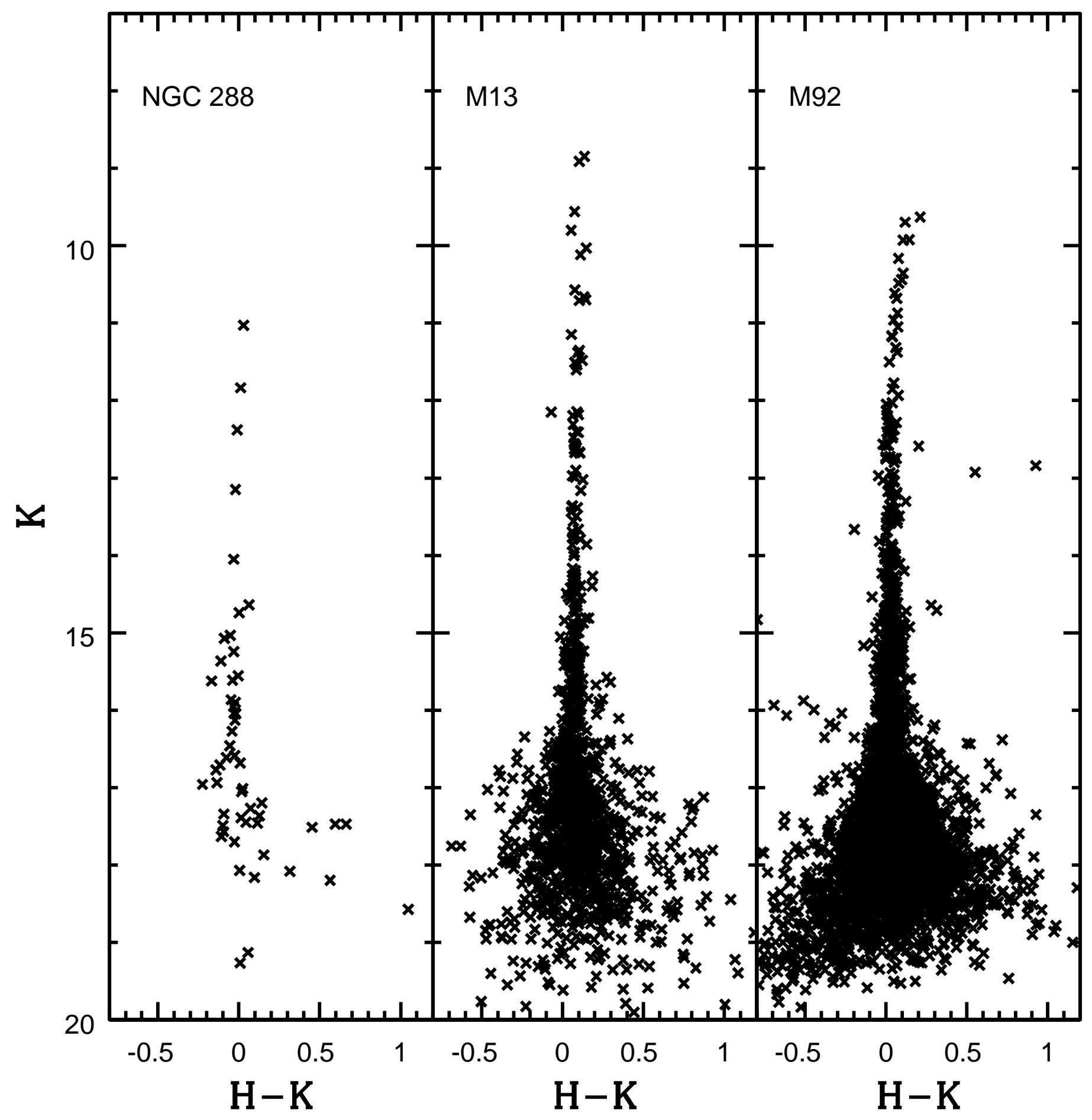




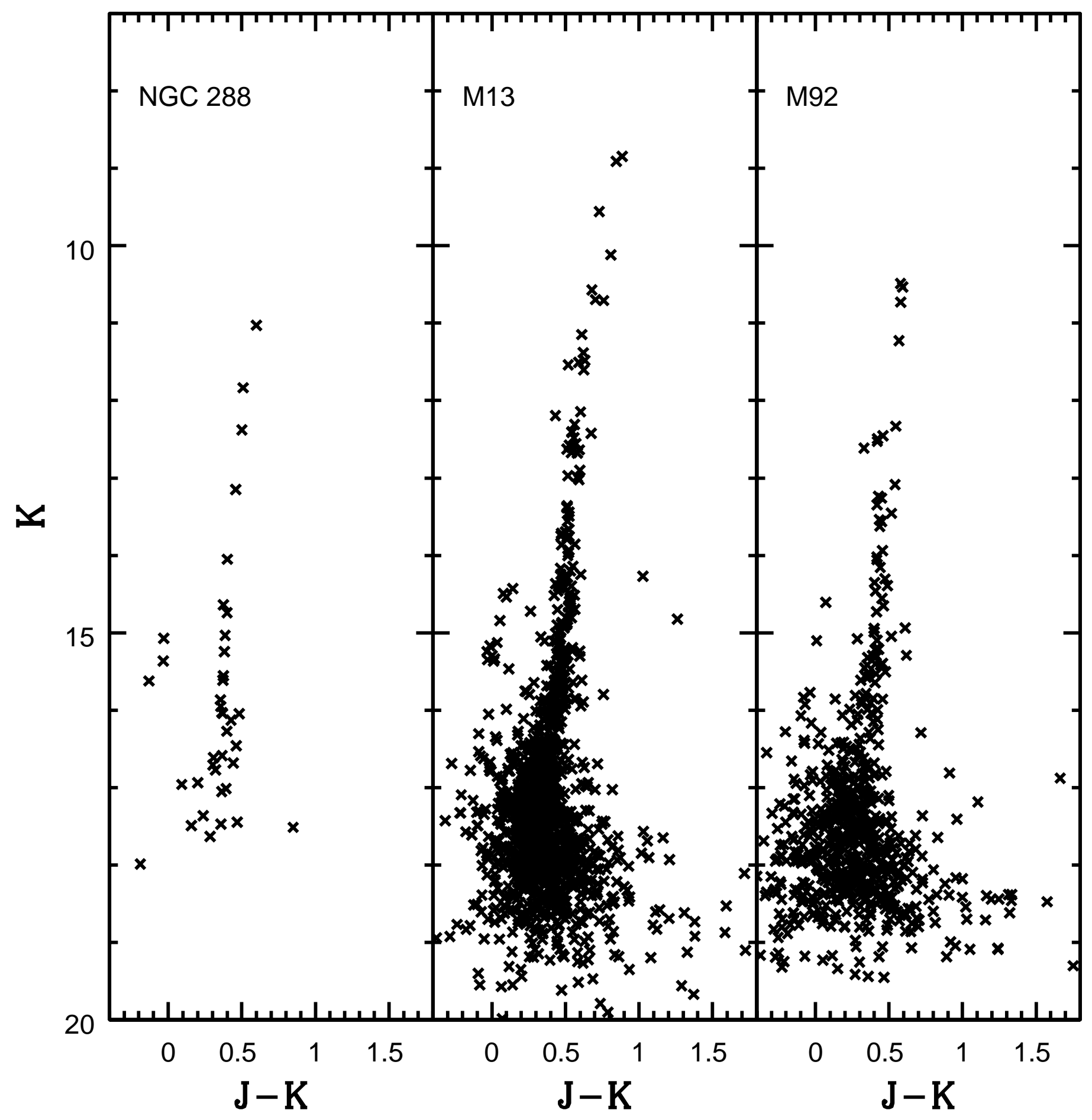




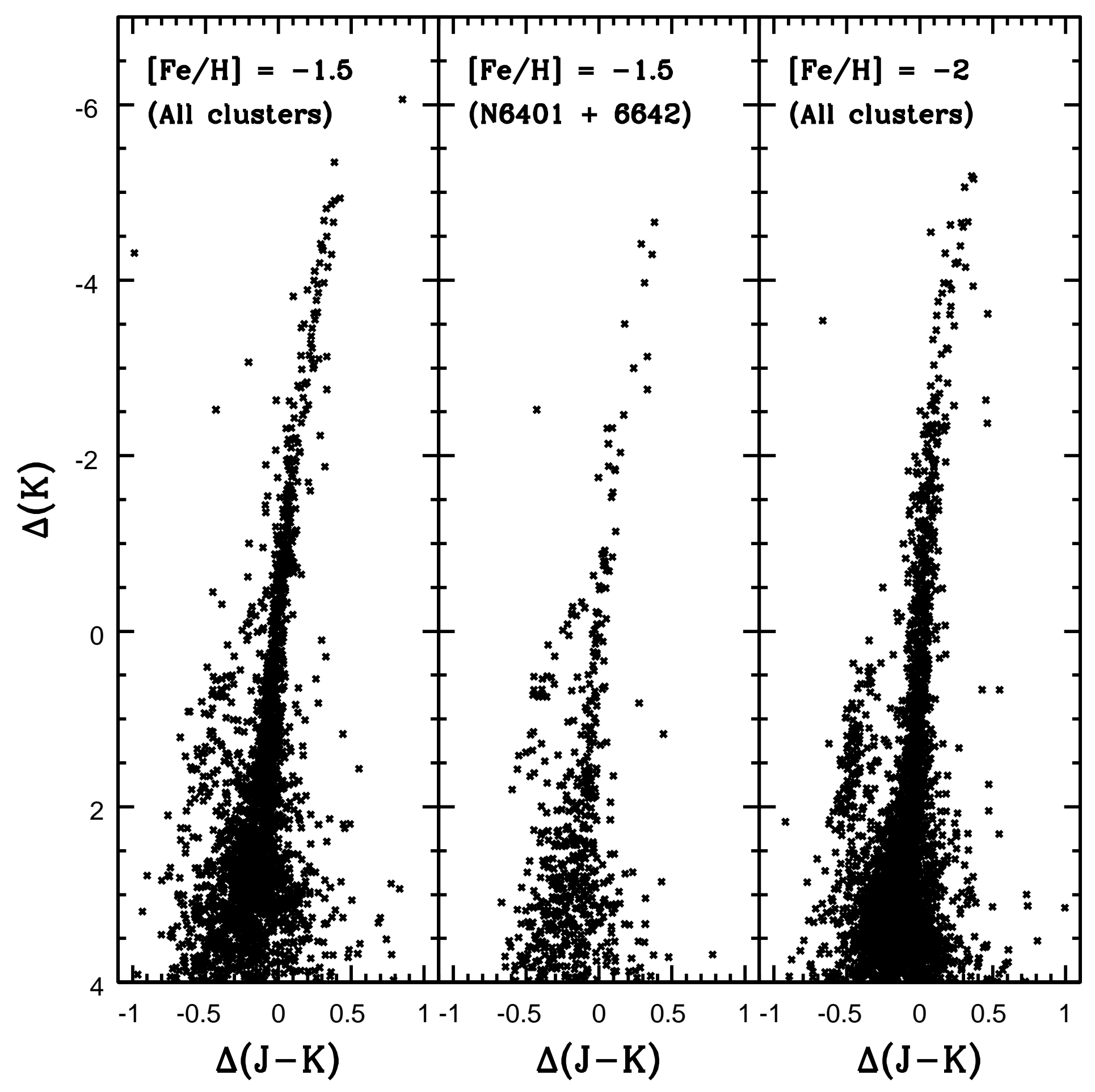




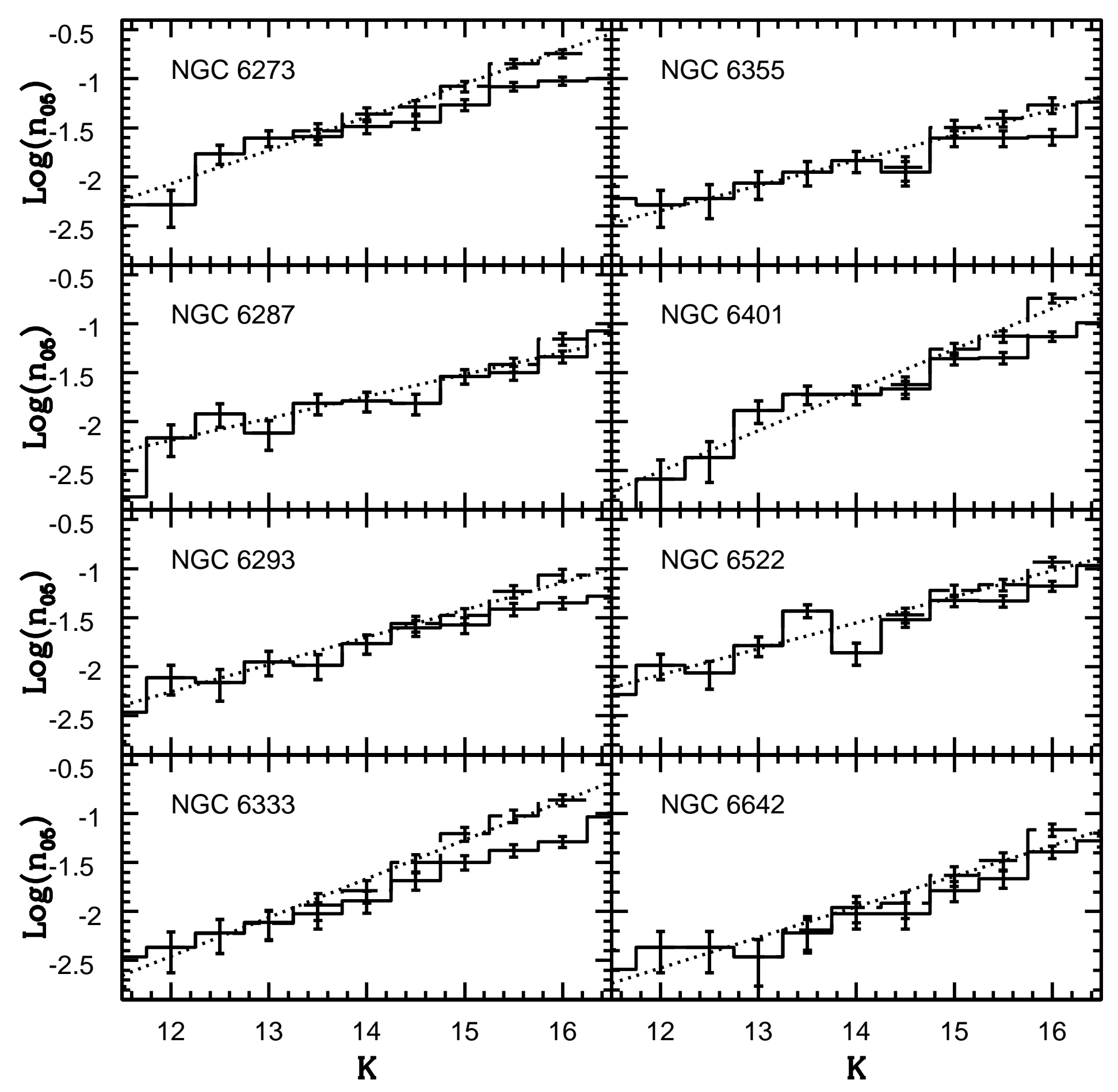




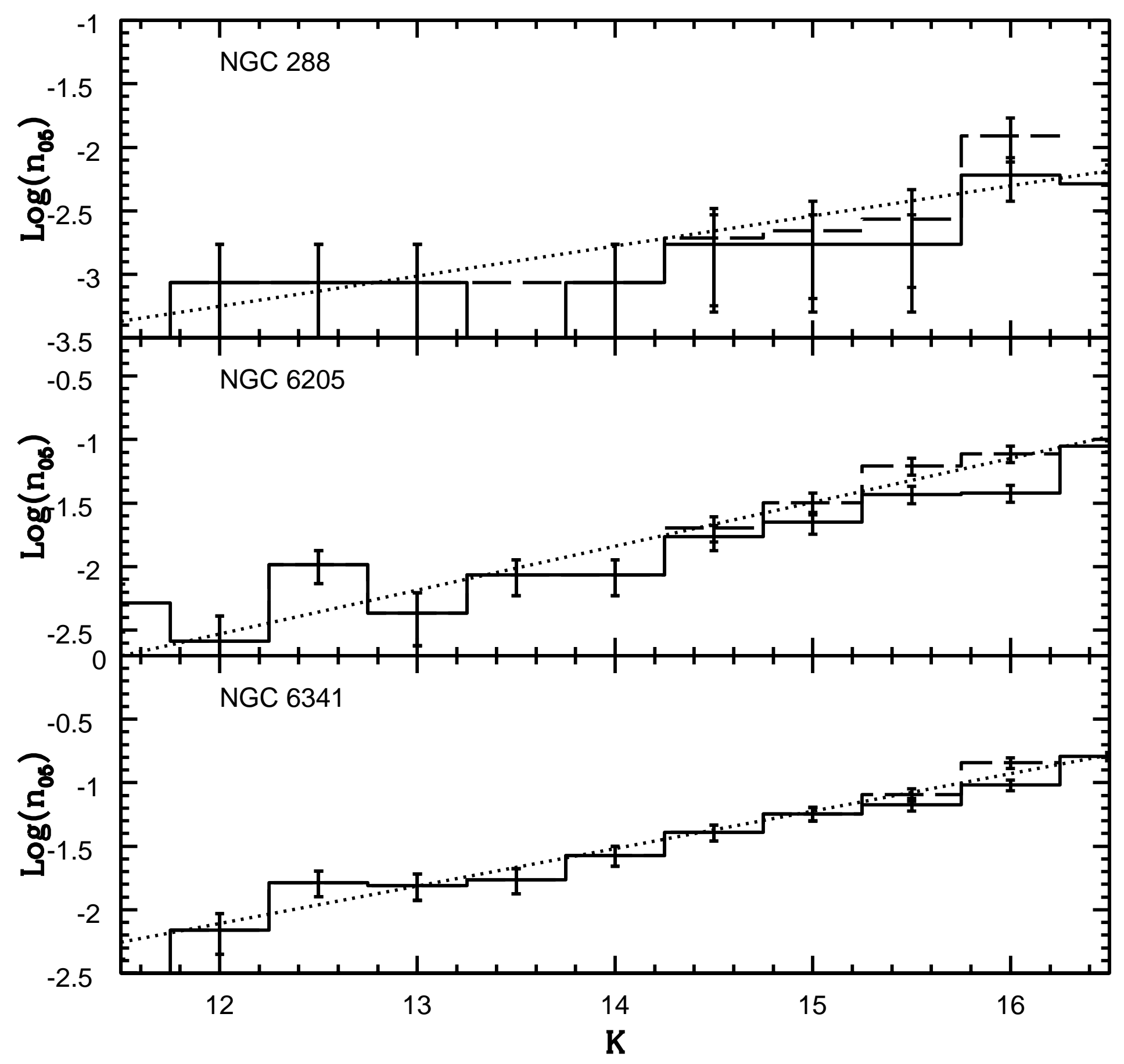




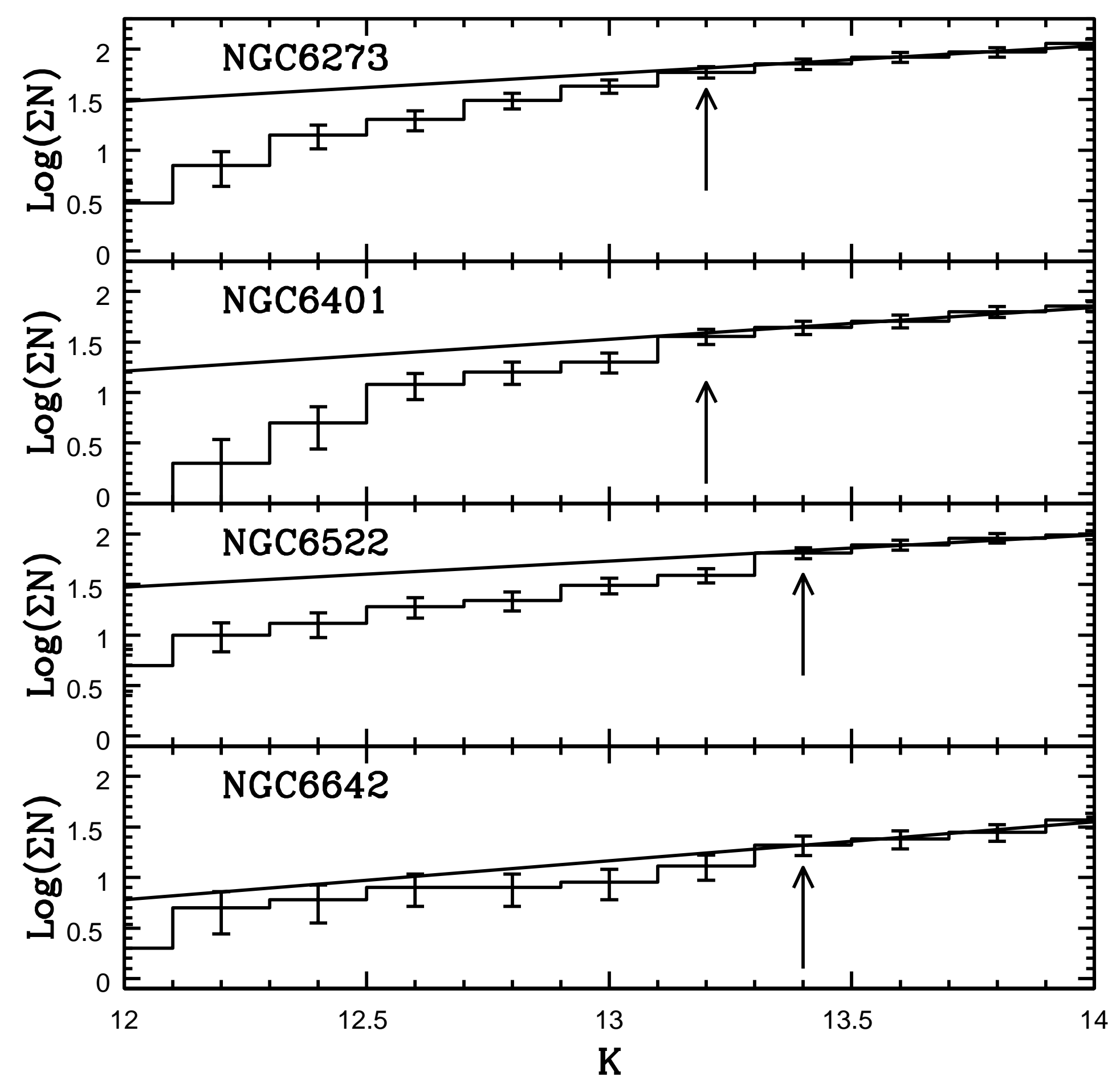




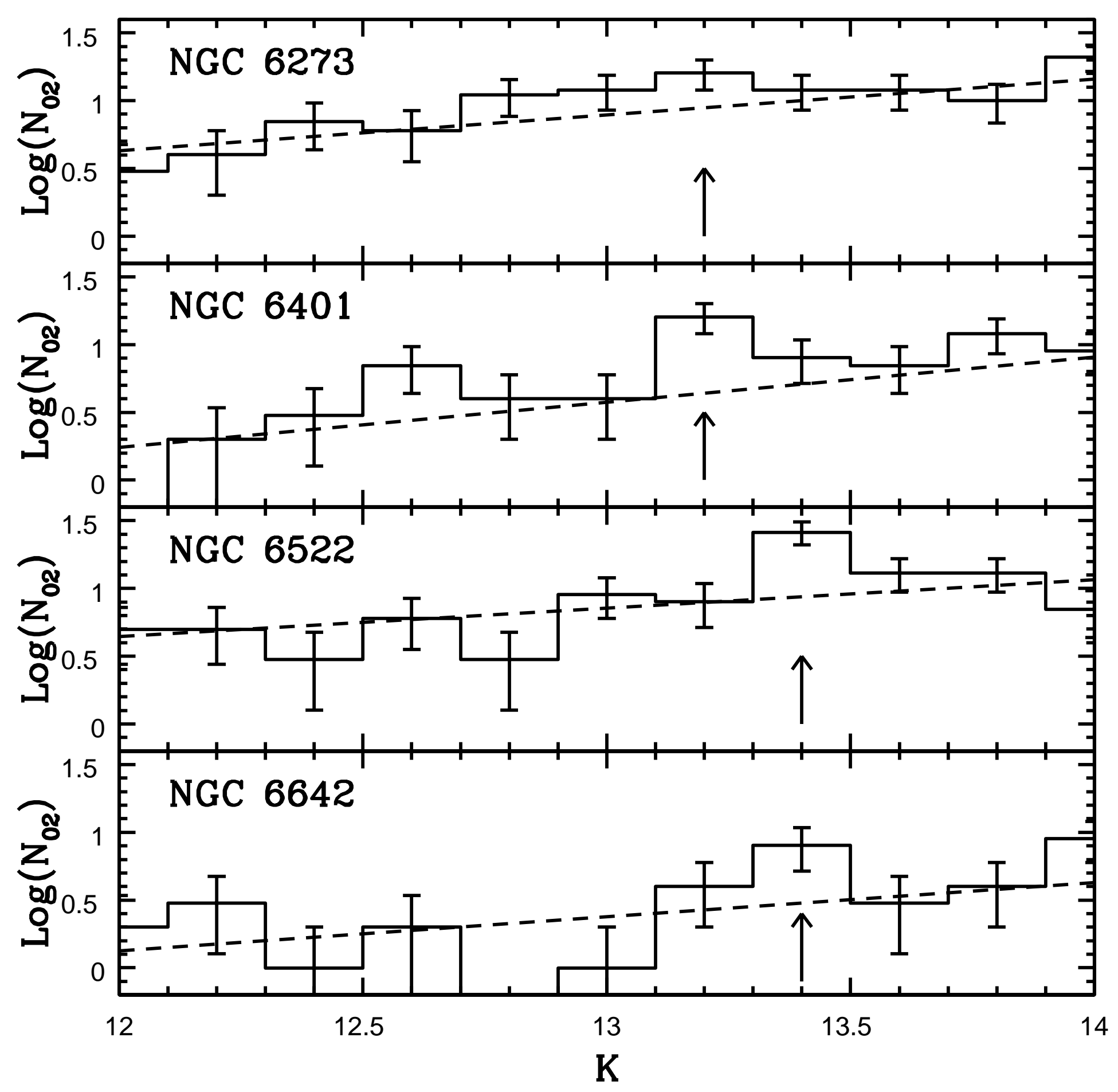

